\title{
New model for datasets citation and extraction reproducibility in VAMDC
}

\author{
Carlo Maria Zwölf*, Nicolas Moreau, and Marie-Lise Dubernet*
}

January 29, 2018

\begin{abstract}
In this paper we present a new paradigm for the identification of datasets extracted from the Virtual Atomic and Molecular Data Centre (VAMDC) e-science infrastructure. Such identification includes information on the origin and version of the datasets, references associated to individual data in the datasets, as well as timestamps linked to the extraction procedure. This paradigm is described through the modifications of the language used to exchange data within the VAMDC and through the services that will implement those modifications. This new paradigm should enforce traceability of datasets, favour reproducibility of datasets extraction, and facilitate the systematic citation of the authors having originally measured and/or calculated the extracted atomic and molecular data.
\end{abstract}

Keywords: database, data citation, atomic data, molecular data

\section{Introduction}

Atomic and Molecular Data Providers are often at the forefront in defining new paradigms for the dissemination of their data to the scientific community:

Since the second half of 1990s, with the spread of the Internet, some historical databases such as VALD [19], HITRAN [23, [24], the Submillimeter, millimeter and microwave spectral line catalog (so-called JPL Catalogue) [18], CDMS [16], 15], GEISA [13], [12] were precursor of a free online sharing of data, thus anticipating of some decades trends later generalised by gouvernements and institutions with the open data and open knowledge initiatives [11], [14, 20].

*LERMA, Observatoire de Paris, PSL Research University, CNRS, Sorbonne University, UPMC Univ Paris 06, 5 Place Janssen, 92190 Meudon, France 
During the last ten years, Atomic and Molecular resources were highly fragmented and available through a variety of interfaces. This situation negatively impacted the full exploitation of the scientific data and has been a major bottleneck between the data providers and the wide body of users. From 2009 to 2014, the goal of the European FP7 projects VAMDC [5] and its successor SUP@VAMDC [27] was to provide the international community with a unique technical and collaborative framework for Atomic and Molecular data sharing: the VAMDC International Consortium [8]. The VAMDC technical e-infrastructure federates, in an interoperable way, a wide range of heterogeneous databases containing data coming from different physical communities, provides a single entry-point both for discovering resources and for accessing the available data in a unified way (http://portal.vamdc.eu). For building and maintaining this e-infrastructure, VAMDC has developed specific procedures, protocols, data formats and query languages (http://standards.vamdc.eu). The Atomic and Molecular data providers community, organised around the VAMDC Consortium, has thus anticipated the identification and resolution of problems that are currently discussed in international groups belonging to the Research Data Alliance (RDA). The goal of RDA is indeed to build the social and technical bridges that enable open sharing of data in the current highly fragmented global research data landscape.

Using the VAMDC facilities, scientists can now easily discover the atomic and molecular resources, and can access in a unique and practical way their contained data. However, the adoption of VAMDC by a large community has revealed a new set of challenging issues. The VAMDC infrastructure data are dynamic. A database exposed through the VAMDC infrastructure may evolve over time: the most recent and precise version of given data may replace old ones as in VALD [19, 25], or new datasets may be added to the already existing ones as in BASECOL 6, 4]. It may happen that some of these database evolutions are not systematically reported through new publications.

Assume then that a scientist extracts from VAMDC at a given time a "dataset", composed of an ensemble of "data", and wishes to use this "dataset" in order to produce some science that will be published into a scientific paper: how can he/she cite this "dataset" and the individual "data"? Since the database content may evolve, for the consistency of the scientific publication, the citation should refer to "datasets" well defined in space (where the "dataset" physically comes from) and time (at what time the "dataset" was produced and extracted). In addition the citations should contain pointers to the authors who originally measured, calculated and/or fitted the individual data. Moreover, for the reproducibility of the scientific process described into the paper referencing the "dataset", everybody wishing to verify step-by step the procedures described into the paper, should be 
able to easily recover the original "dataset" and replay the data-production workflow.

In spring 2014 we joined the RDA Data Citation Working Group in order to study these open questions. The goal of this Working Group [22] is to create the identification mechanisms that

- allows us to identify and cite arbitrary views of a "dataset" in a precise, machine actionable manner;

- allows us to cite and retrieve that "dataset" as it existed at a certain point in time, whenever the database is static or highly dynamic;

- is stable across different technologies and technological changes.

The very new model we will discuss through this paper is the mechanisms we may apply on the data coming from the VAMDC e-infrastructure in order to meet the recommendations of the RDA Working Group on Data Citation with the goal of obtaining

- a rigorous and reproducible data citation model,

- the reproducibility of data production (or data processing) workflows,

and in order to facilitate the citation of the original authors of the atomic and molecular data.

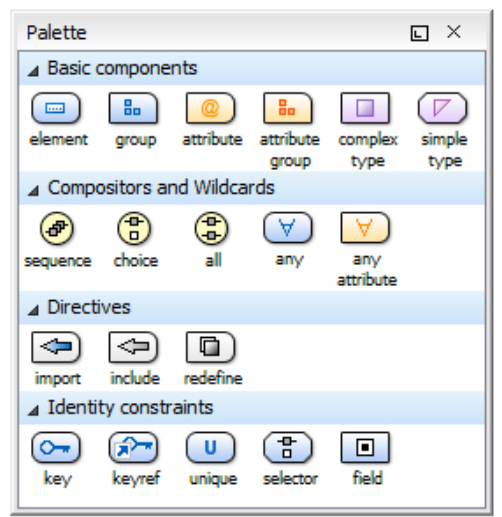

Figure 1: Graphical convention adopted for building graphical representations, starting from the XML schema.

\section{Existing VAMDC solution for data citation}

All the output of the VAMDC infrastructure are built upon an abstract object model: each specific output is an instance of this model. For historical 


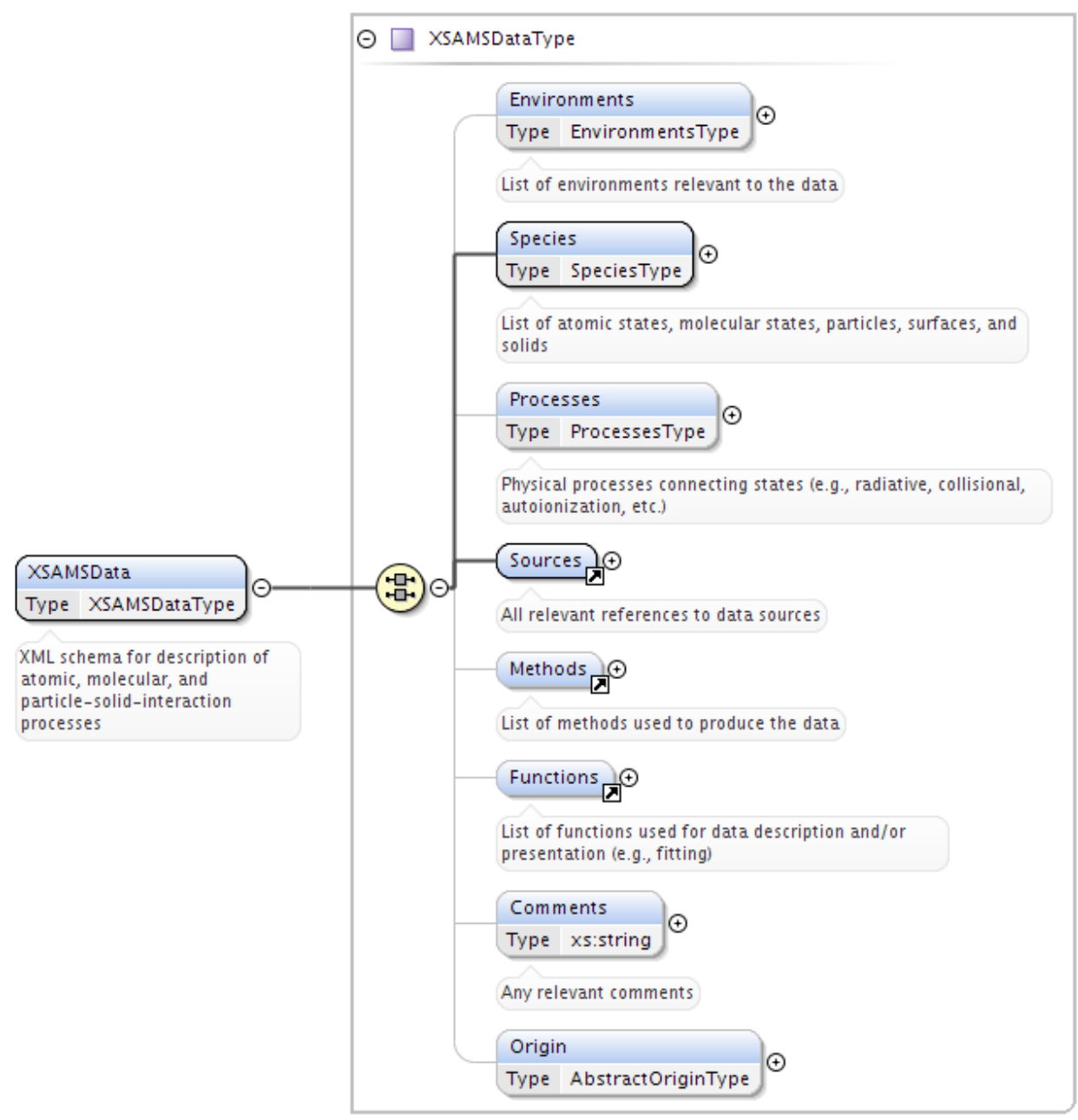

Figure 2: Representation of the root element of an XSAMS file. 
reasons, without loss of generality, the model has been fixed into an XML format, the XSAMS XSD schema ${ }^{1}$.

\subsection{Graphical convention for representing XML elements}

The graphical diagrams displayed in this document are a simple rendering of every XML element contained in the XSAMS, and they are obtained following the graphical conventions of Fig. 1. In this graphical representation (see Fig. 2 for example) every complex element is linked with segments to the contained sub-elements. A bold segment indicates that the sub-element is required and a thin segment indicates that the sub-element is optional. Moreover, the cardinality of the contained sub-elements can be expressed on the segments. The detailed explanation of the XSAMS components can be found in the official VAMDC XSAMS documentation at http://www.vamdc.org/standards.

\subsection{Example of data-citation need for scientific authors}

Surveys of interstellar regions requires the use of spectroscopic information within the observed range of wavelengths/frequencies. As an example, the survey by [10] covers frequencies from $83302 \mathrm{MHz}$ to $262404 \mathrm{MHz}$ and detect emission from about 36 species. For that survey, [10] indicate that they used catalogues from two public databases [18, [15] and one private database of J. Cernicharo (private communication). We note that there is no knowledge of the exact dataset used in the analysis, and therefore the analysis may not be reproductible if the database contents evolve over the years. Secondly, we note there is no citation of the authors who produced the spectroscopic data. Obviously for such large surveys with so many species there is a large contribution from many experimental/theoretical spectroscopic papers. On the contrary, that for the non-local thermodynamical equilibrium analysis of spectra (that includes the use of collisional rate coefficients) about 12 publications related to collisional data are cited. This dichotomy of treatment could be first explained by the complexity of citing/finding many spectroscopy authors, while it is easy to cite a few collisional papers.

Similarly, another survey [2] cites many spectroscopic databases without citing either the original authors or the version of data used in the survey's analysis. The study of Punanova et al. 21] cites the authors of transitions that are not part of a database, such as the hyperfine transitions of $\mathrm{N}_{2} \mathrm{H}^{+}$ [17] and such as the $1 \rightarrow 0$ transition of $\mathrm{C}^{17} \mathrm{O}$ [9], but they cite the splatalogue catalog (http://www.cv.nrao.edu/php/splat/) for the $1 \rightarrow 0$ transition

\footnotetext{
${ }^{1}$ XSAMS is the acronym for: XML Schema for Atoms, Molecules and Solids
} 
of $\mathrm{C}^{17} \mathrm{O}$. Those examples confirm that the origin and versioning of spectroscopic data might be lost along the chain of usage as soon as spectroscopic data are taken from a database.

Through the VAMDC e-infrastructure, the protocols presently allow to retrieve the references associated to the spectroscopic and collisional data in a straightforward and quick manner. For example, from the result page of the VAMDC portal (cf. figure 3) the "bibtex" visualisation processor allows one to get references attached to the data in a usable form for publication. This processor produces XSAMS output file (produced by the VAMDC infrastructure) by extracting the bibliographic information contained into the Sources field, which is embedded into each data entry (cf. figure 2 and (4). Thus, the use of the current version of VAMDC allows full citation of any data and such citation relies on the good will of the users. Even so, the citation process may become cumbersome when the extracted datasets come from many sources.

This latter issue and the former issue of reproducibility of the extraction process are addressed in the new paradigm of datasets citation that we describe below.

\section{Introducing the evolutions of the XSAMS for- mat}

In this paragraph we discuss new mechanisms allowing one to identify through time and location, in a sustainable and unique way, the data sets extracted by users from the VAMDC e-infrastructure. The proposed mechanisms comes with an evolution of the XSAMS standard.

The practical usage of the described features is presented in section 5

\subsection{Introduction of the new schema elements for handling data versioning and origin}

The root element of a XSAMS file is the XSAMSData object (cf. figure 2), which is an instance of the XSAMSDataType class. The innovation compared with the current official version of the XSAMS format (version 12.07) is the introduction of the Origin field, that contains the new mechanisms for handling the datasets citations.

The Origin field is an instance of a class extending the AbstractOriginType (cf. figure 5). This abstract type must contain:

- a unique Timestamp. This field defines the exact time when the data have been extracted by the infrastructure; 


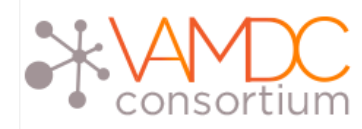

Home VAMDC databases Guided query Advancedquery Saved queries I Disclaimer Citation policy Info Feedback

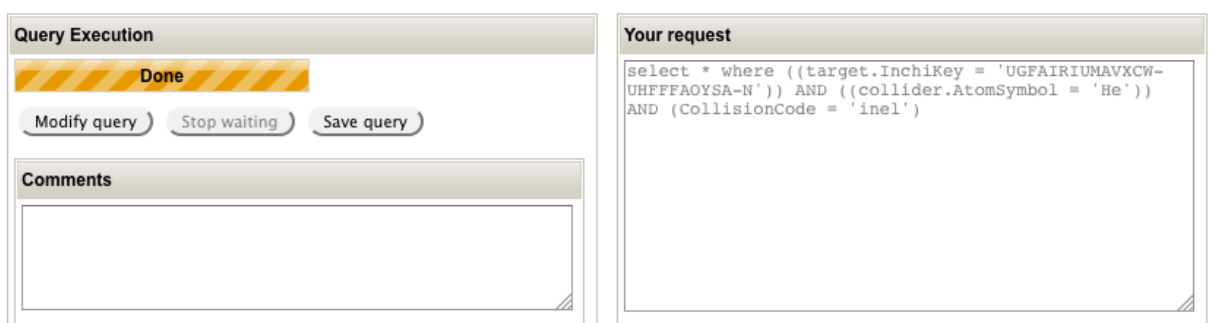

\begin{tabular}{|c|c|c|c|c|c|c|c|}
\hline \multicolumn{8}{|l|}{ Results by node } \\
\hline Name & View data & Response & $\frac{\text { Last database }}{\underline{\text { update }}}$ & Download & Species & States & Processes \\
\hline \multirow[t]{2}{*}{ BASECOL: VAMDC-TAP interface } & -- Choose display -- & OK & 01/07/2005 & XSAMS & 6 & 0 & 2 \\
\hline & \multirow{4}{*}{\multicolumn{2}{|c|}{$\begin{array}{l}\text {-- Choose display -- } \\
\text { ** Collisional data XSAMS to HTML } \\
\text { ** BibTeX from XSAMS } \\
\text { ** XSAMS multiplexor } \\
\text { Table views of XSAMS } \\
\text { Xsams2SME } \\
\text { Atomic spectroscopy XSAMS to HTML } \\
\text { Molecular spectroscopy XSAMS to HTML } \\
\end{array}$}} & & & & & \\
\hline KIDA: VAMDC-TAP interface & & & $\begin{array}{c}19 / 09 / 2011 \\
10: 44\end{array}$ & XSAMS & 3 & 0 & 0 \\
\hline RADAM - Ion Interactions & & & Not available & & 0 & 0 & 0 \\
\hline $\begin{array}{l}\text { IDEADB - Innsbruck Dissociative Electron } \\
\text { Attachment Database }\end{array}$ & & & Not available & & 0 & 0 & 0 \\
\hline
\end{tabular}

Figure 3: Detail of the result page of the VAMDC portal highlighting the "bibtex" processor. 


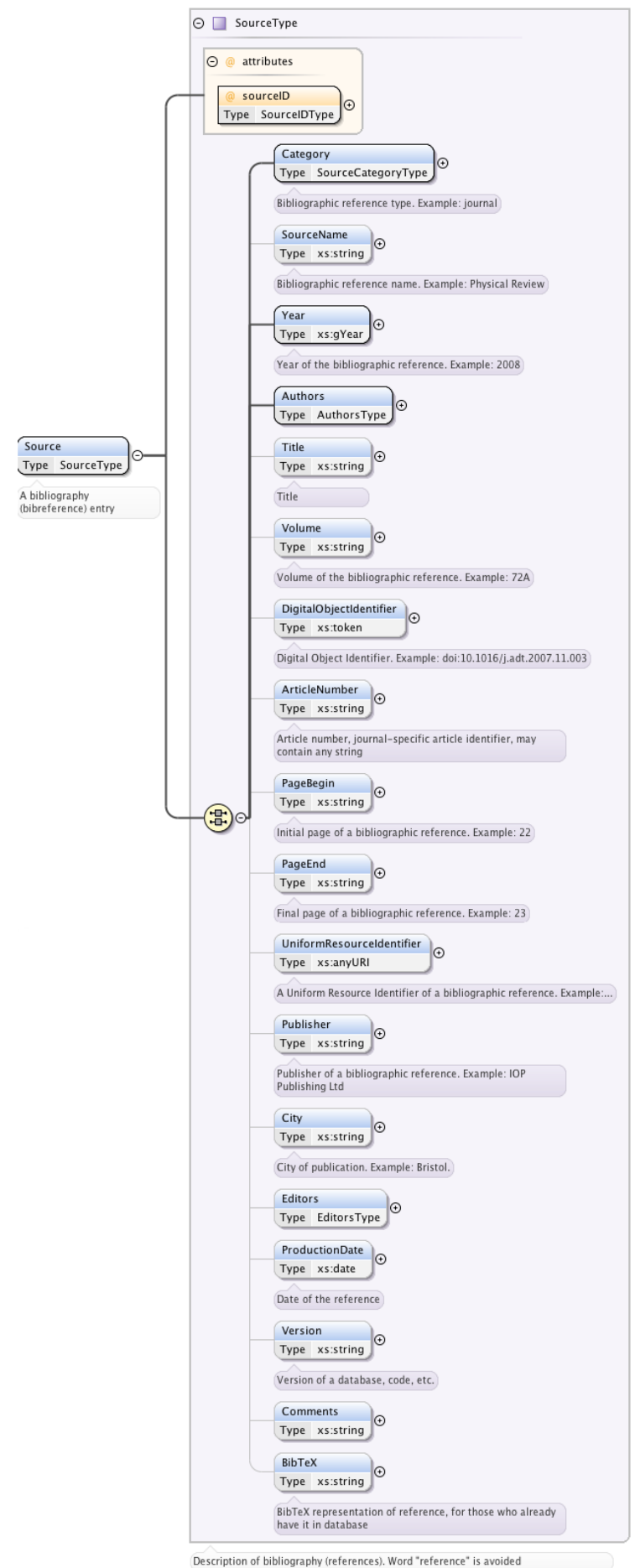

Figure 4: Details of the Source field contained in XSAMS files. 


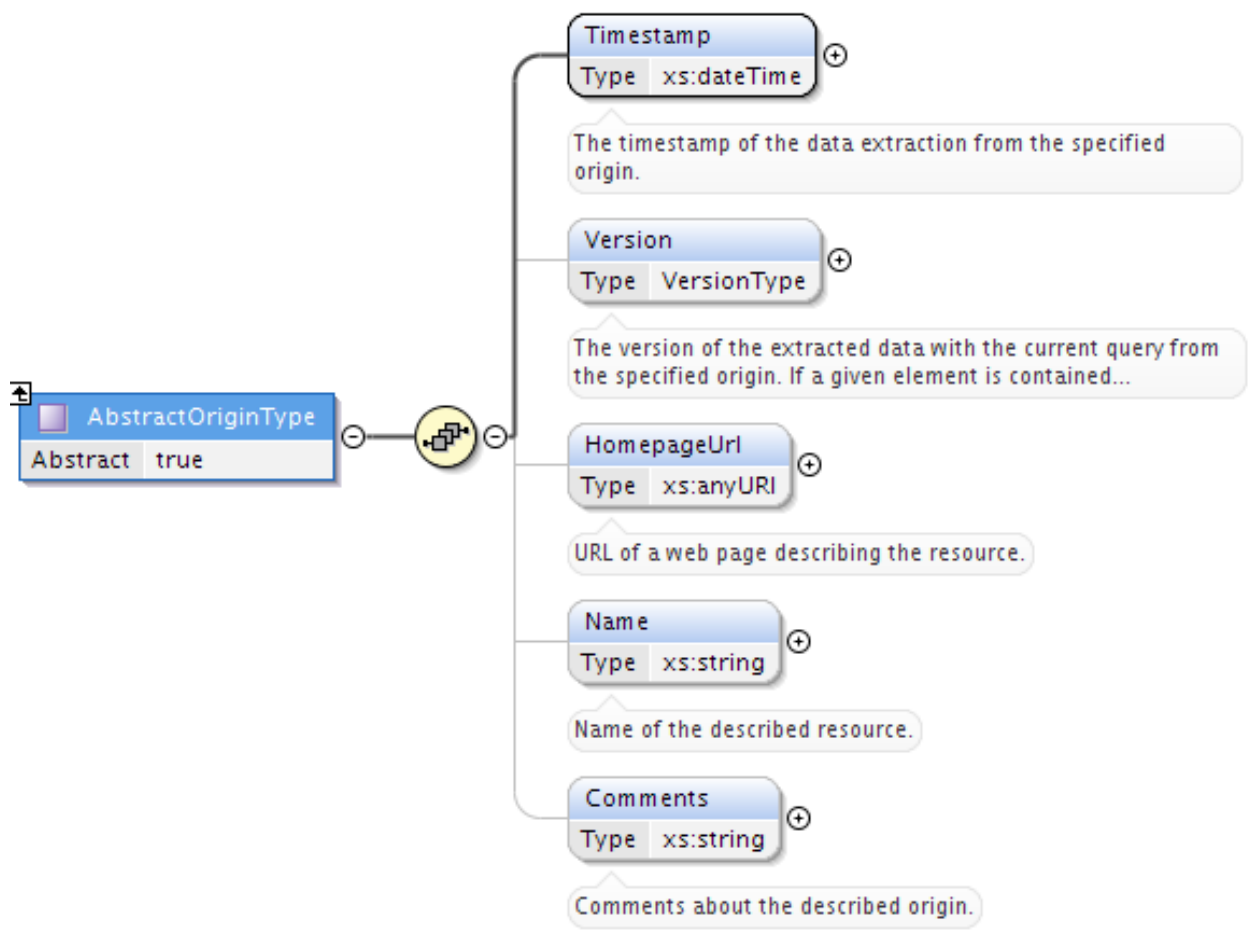

Figure 5: Representation of the abstract element AbstractOrigin Type. 
- a Version field, instance of VersionType class (cf. paragraph 3.1.1);

- a HomepageURL field, which is the link to the website describing the resource that produced the data;

- a Name field, which is the name of the resource producing the data;

- a Comments field, which (as its name indicates), contains the comments related with the resources producing the data.

The three classes extending the abstract type AbstractOrigin Type are: VamdcNodeOrigin Type, VamdcProcessorOrigin Type, and OtherOriginType.

The VamdcNodeOrigin Type (cf. figure 6), as its name indicates, is used when the resource producing the data is a VAMDC node. It extends the AbstractOrigin Type by mandatorily adding:

- a Query field, which is a text string defining the query used for extracting the data from the described node.

- an OriginIdentifier field, which is the identifier of the node into the VAMDC infrastructure, as it appears into the VAMDC registry.

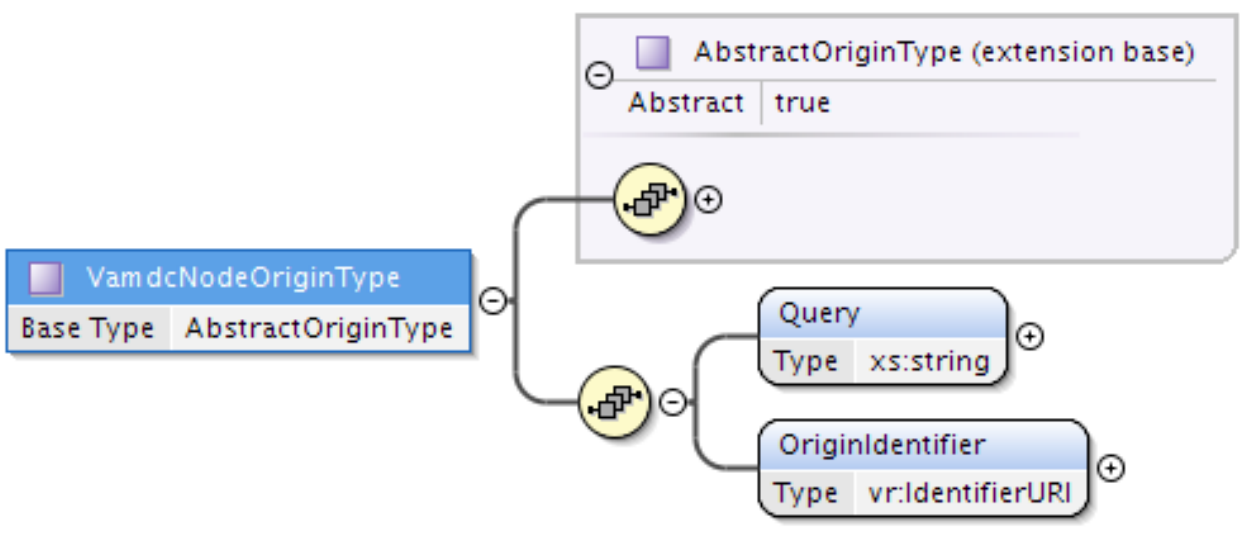

Figure 6: Representation of the element VamdcNodeOriginType.

The VamdcProcessorOriginType (cf. figure 7), as its name indicates, is used when the resource producing the data is a VAMDC processor. It extends the AbstractOrigin Type by mandatorily adding:

- an OriginIdentifier field, which is the identifier of the processor into the VAMDC infrastructure, as it appears into the VAMDC registry. 
- the list (which must contain at least one element) of the Origins that produced the data used as inputs by the current processor.

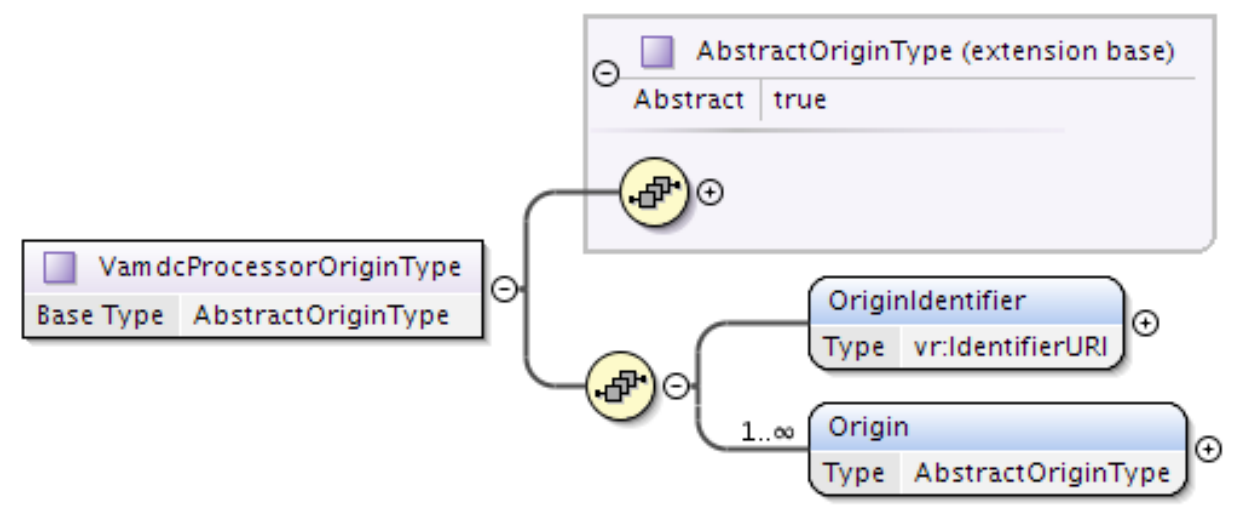

Figure 7: Representation of the element VamdcProcessorOriginType.

The OtherOrigin Type (cf. figure 8), as its name indicates, is used when the resource producing the data is not a VAMDC node and neither a VAMDC processor. It extends the AbstractOrigin Type by adding:

- an OriginIdentifier field, which is the identifier of the resource into the VAMDC infrastructure, as it appears in the VAMDC registry.

- the list of the Origins that produced the data used as inputs by the current resource.

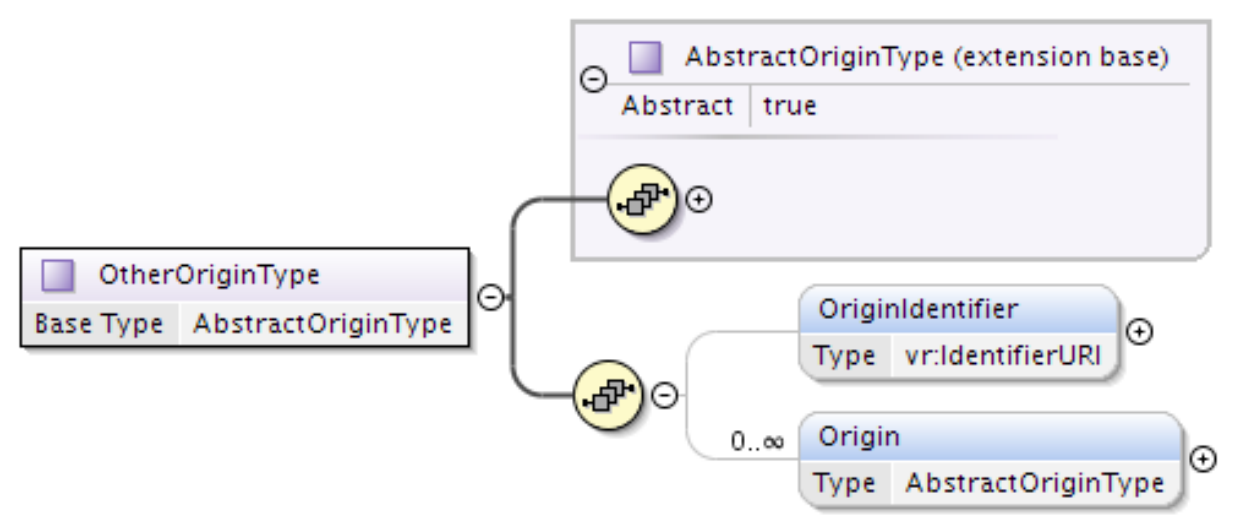

Figure 8: Representation of the element OtherOriginType. 


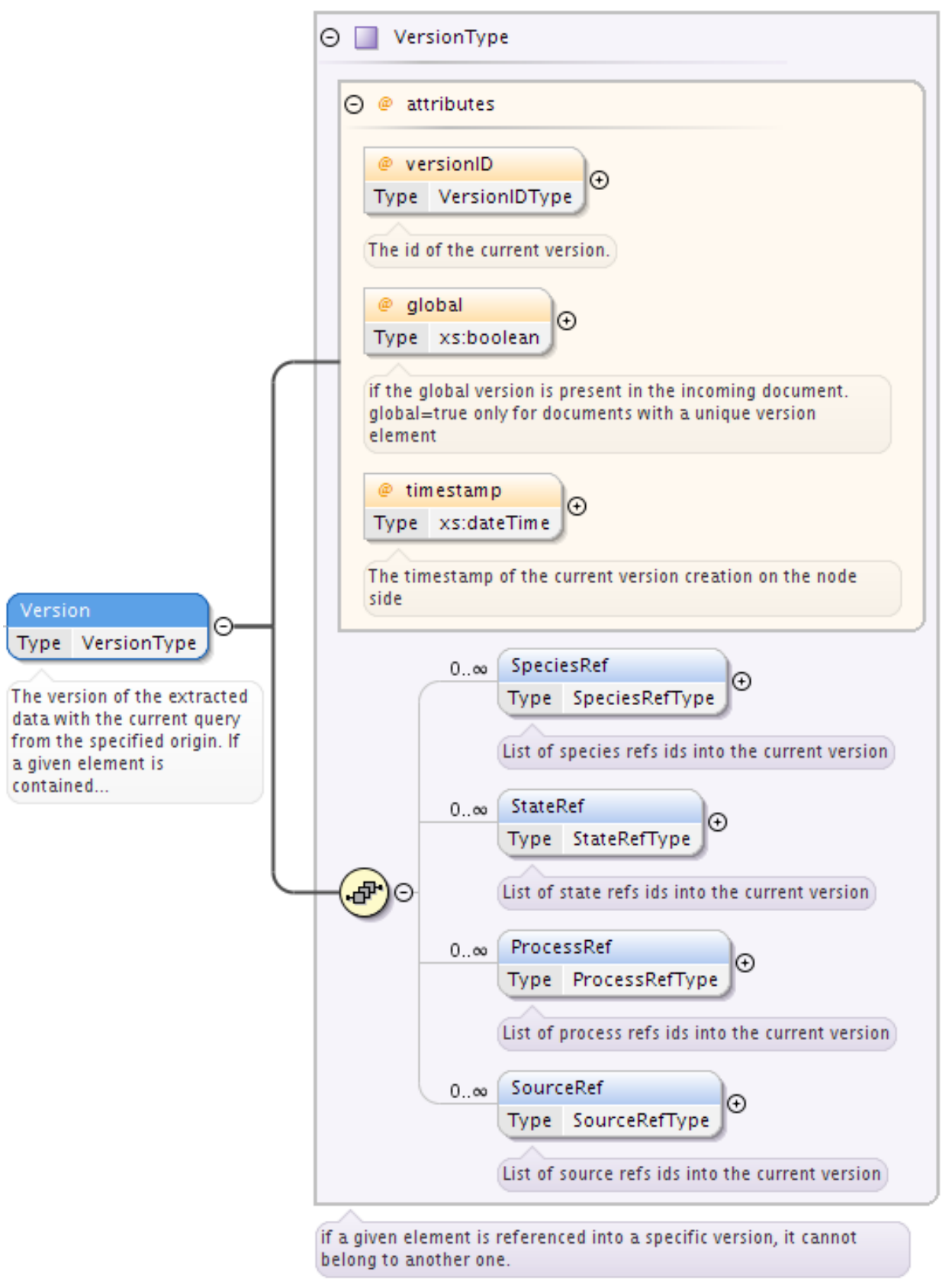

Figure 9: Representation of the element VersionType. 
These three classes make sure that the users know what is the latest treatment of the data, and what are the original sources of the data, thus making possible to trace the original public exposure of the data.

\subsubsection{The Version element}

With the Version Type class the data contained in a XSAMS file are managed with fine grained granularity (cf. figure 9). This class contains three attributes:

- a versionID which is the unique identifier of the current version instance;

- a global boolean field. If global is true, then XSAMS file should consist of a unique instance of VersionType and all the data contained into the file will automatically belong to this Version. This implies that the attribute global equal to true is legal for XSAMS document with a unique Origin element (since Origin automatically contains a Version).

- a unique timestamp field which define the timestamp of the current version creation on the resources providing the data.

Moreover, this class contains three lists of elements:

- a list of SpeciesRef objects of type SpeciesRefType. In other words, this list contains the references to all the species (defined elsewhere into the XSAMS file) which belong to the current Version;

- a list of StateRef object of type StateRefType. This list contains the references to all the states (defined elsewhere into the XSAMS file) which belong to the current Version;

- a list of ProcessRef object of type ProcessRefType. This list contains the references to all the process (defined elsewhere into the XSAMS file) which belong to the current Version.

- a list of SourceRef object of type SourceRefType. This list contains the references to all the bibliography sources (defined elsewhere into the XSAMS file) which belong to the current version.

If a given element is referenced into a specific Version object, it can not belong to another one.

Remark: It is important to underline the difference between the timestamp field contained into an Origin object and the timestamp contained into a Version object. The first corresponds to the time when the data were extracted by the user (i.e. the data when the file was created) while the second corresponds to the time when that specific version of data was made available to community by the resource providing the data. 


\section{Some examples of data extracted from VAMDC, formatted with the proposed schema}

In this paragraph we will provide examples of data extracted from the VAMDC infrastructure and formatted with the schema described in the previous section. For reason of clarity, we have lightened those examples, by manually selecting only a subset of the data really available into VAMDC. This will not affect the methods and the accuracy of the described approach.

The VAMDC internal convention is to use wavelength expressed in angstrom when querying radiative data from databases. This is imposed by the need of interoperability within VAMDC. Nevertheless from our portal ([8], http://portal.vamdc.eu) the users may query the radiative databases using whatever relevant quantities (frequency, energy, wavenumber, wavelength) associated to a choice of relevant units. No change in the original data is performed in the extracted output. For example B, line 108 shows that CDMS provides frequencies in $\mathrm{MHz}$.

\subsection{Example of an extraction from the BASECOL node}

Let us consider an elastic collisional process, where the target is $C O$ and the collider is $H e$. Let us try to find some data on this collision into the VAMDC infrastructure. The associated query (generated by the portal from the Graphical User Interface and passed to all the VAMDC nodes) is:

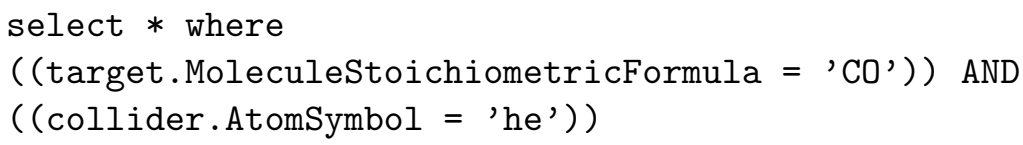

The file reported in its entirety in $\mathrm{A}$ is the output that the BASECOL node 4 generates when answering the previous query (with the reservations made at the beginning of paragraph 4). Let us browse the content of this file and focus on the Origin element:

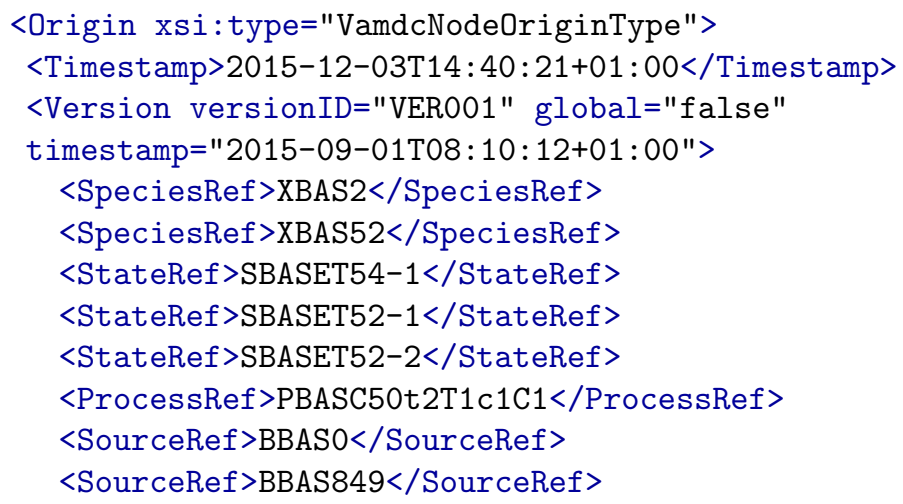




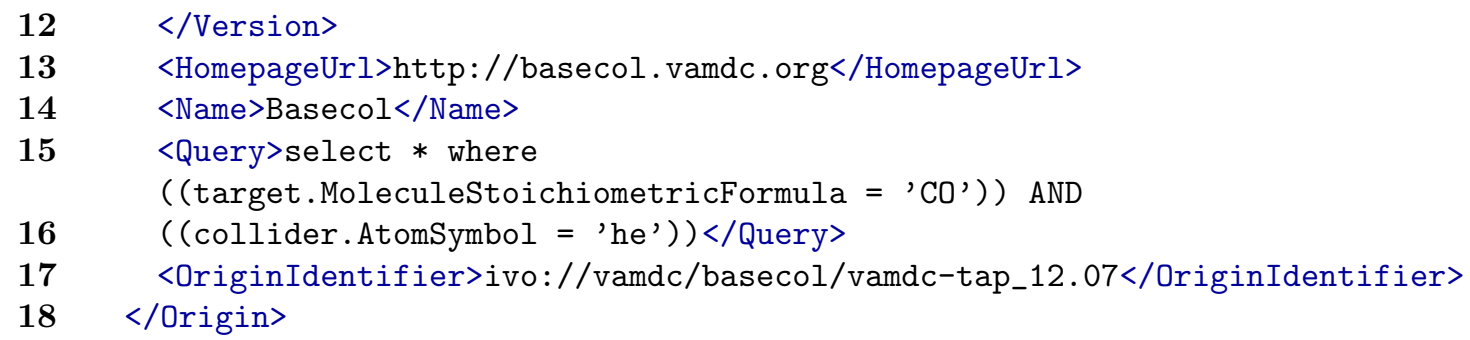

The first line indicates that the resource producing the file is a VAMDC node and the second line indicates when the extraction has been performed (this time also corresponds to the creation of the file). On lines 3 to line 12 we find the information about the versioning of the contained data: it is specified that

- the species referenced by the identifiers XBAS2, XBAS52 (defined respectively at lines 56 and 89 of $\mathrm{A}$;

- the states referenced by the identifiers SBASET54-1, SBASET52-1, SBASET52-2 (defined respectively at lines 58, 119 and 134 of A);

- the processes referenced by the identifier PBASC50t2T1c1C1 (defined at line 154 of A

- the sources referenced by the identifiers BBASO, BBAS849 (defined respectively at line 194 and 207 of $\mathrm{A}$.

belong to the version VER001 of the database. This version was made available on the date 2015-09-01T08:10:12+01:00.

Starting from line 13 we have the information about the database that produced the data: the link to its homepage (line 13), its Name (line 14), the query used for generating extracting the data and generating the file (lines 15 to 16), the unique identifier of the resource as it appears into the VAMDC infrastructure registry (line 17).

We recall that the two bibliographic references $B B A S 0$ and $B B A S 849$ included into this VER001 version (cf. the last bullet of the previous item list) are respectively defined at line 194 of $\mathrm{A}$ :

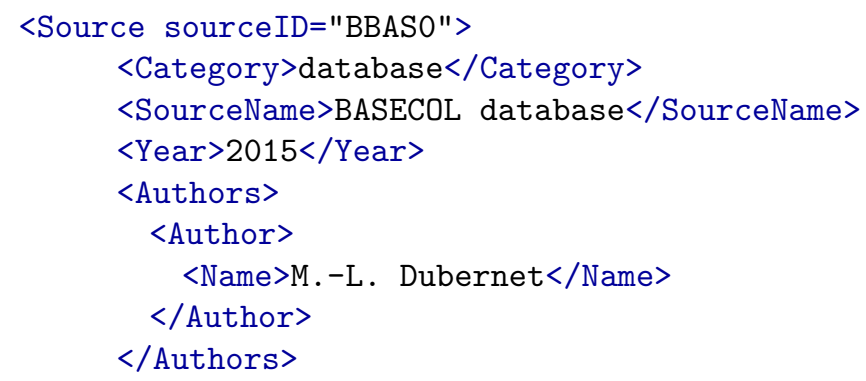




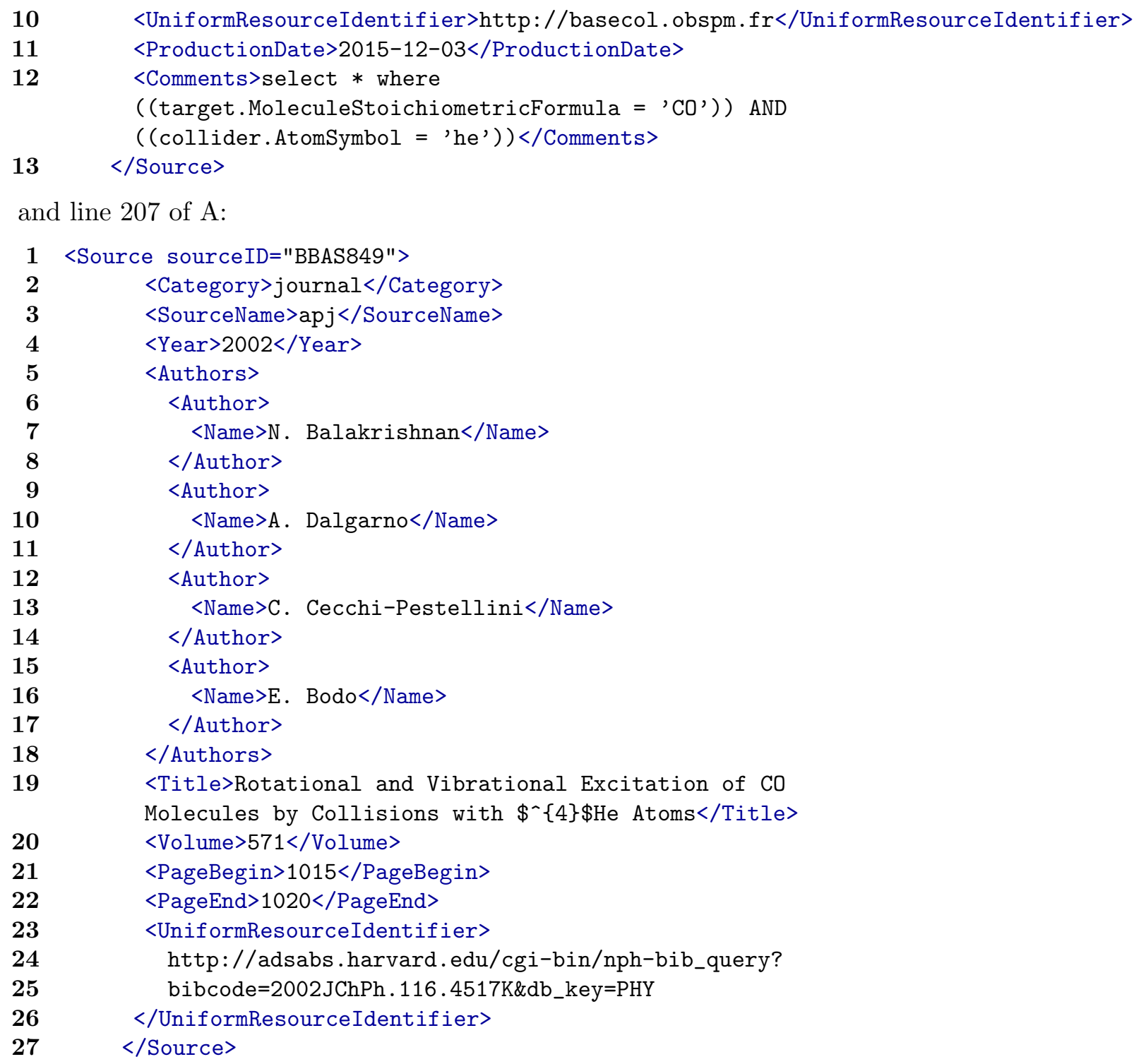

This last reference is [1]

\subsection{Example of an extraction from the CDMS node}

Let us now try to extract from the VAMDC infrastructure some spectroscopic data for the carbon monoxyde in a range of wavelength between $2.600610^{7} \AA$ and $2.600810^{7} \AA$. The associates query (typically generated by the portal from the Graphical User Web Interface and passed to all the VAMDC nodes) is

select $*$ where

(RadTransWavelength >= 2.6006E7 AND 
RadTransWavelength $<=2.6008 \mathrm{E} 7)$ AND

$(($ MoleculeStoichiometricFormula $=$ 'CO'))

The file reported in its entirety in $\mathrm{B}$ is the output that the CDMS node [15] generates when answering the previous query (with the reservations made at the beginning of paragraph 44. Let us browse the content of this file and focus on the Origin element:

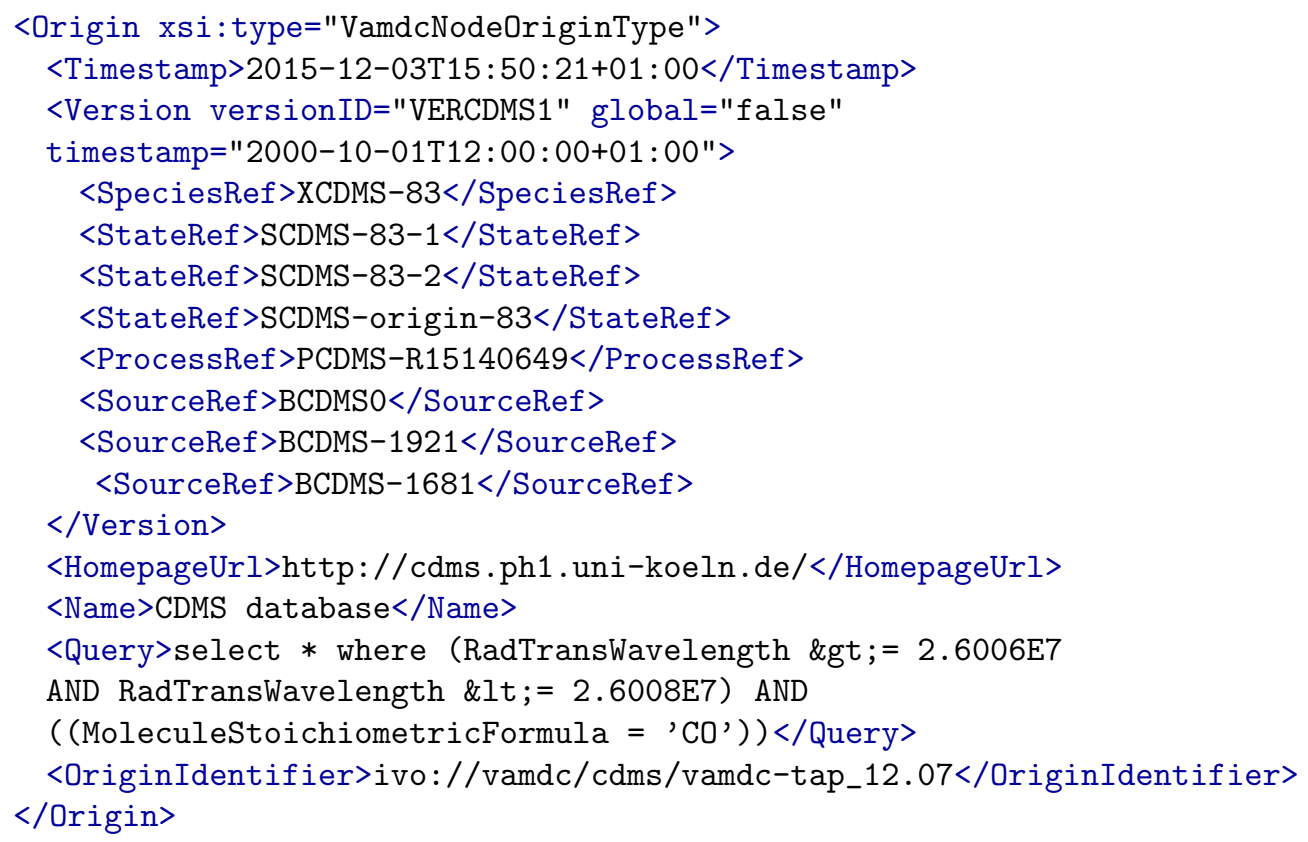

As in the example from BASECOL, the first line indicates that the resource producing the file is a VAMDC node and the second line indicates when the file was generated. From line 3 to 12 we find the information about the versioning of the contained data: it is specified that

- the species referenced by the identifier $X C D M S-83$ (defined at line 24 of B);

- the states referenced by the identifiers $S C D M S-83-1, S C D M S-83-2$, $S C D M S$-origin-83 (respectively defined at lines $67,83,51$ of $\mathrm{B}$ );

- the process referenced by the identifier PCDMS-R15140649 (defined at line 105 of B

- the sources referenced by the identifier BCDMSO, BCDMS-1921, BCDMS1681 (defined respectively at line 131, 150 and 170 of $B$ )

belong to the version VER1 of the database. This version was made available on the date 2000-10-01T12:00:00+01:00.

Starting from line 13 we have the information about the database that produced the data: the link to its homepage (line 13), its name (line 14), the 
query used for extracting the data and generating the file (line 13), the unique identifier of the resource, as it appears into the VAMDC infrastructure registry (line 15).

We recall that the three bibliographic references $B C D M S 0, B C D M S$ 1921 and $B C D M S-1681$ (cf. the last bullet of the previous item list) are respectively defined at line 131 of $B$

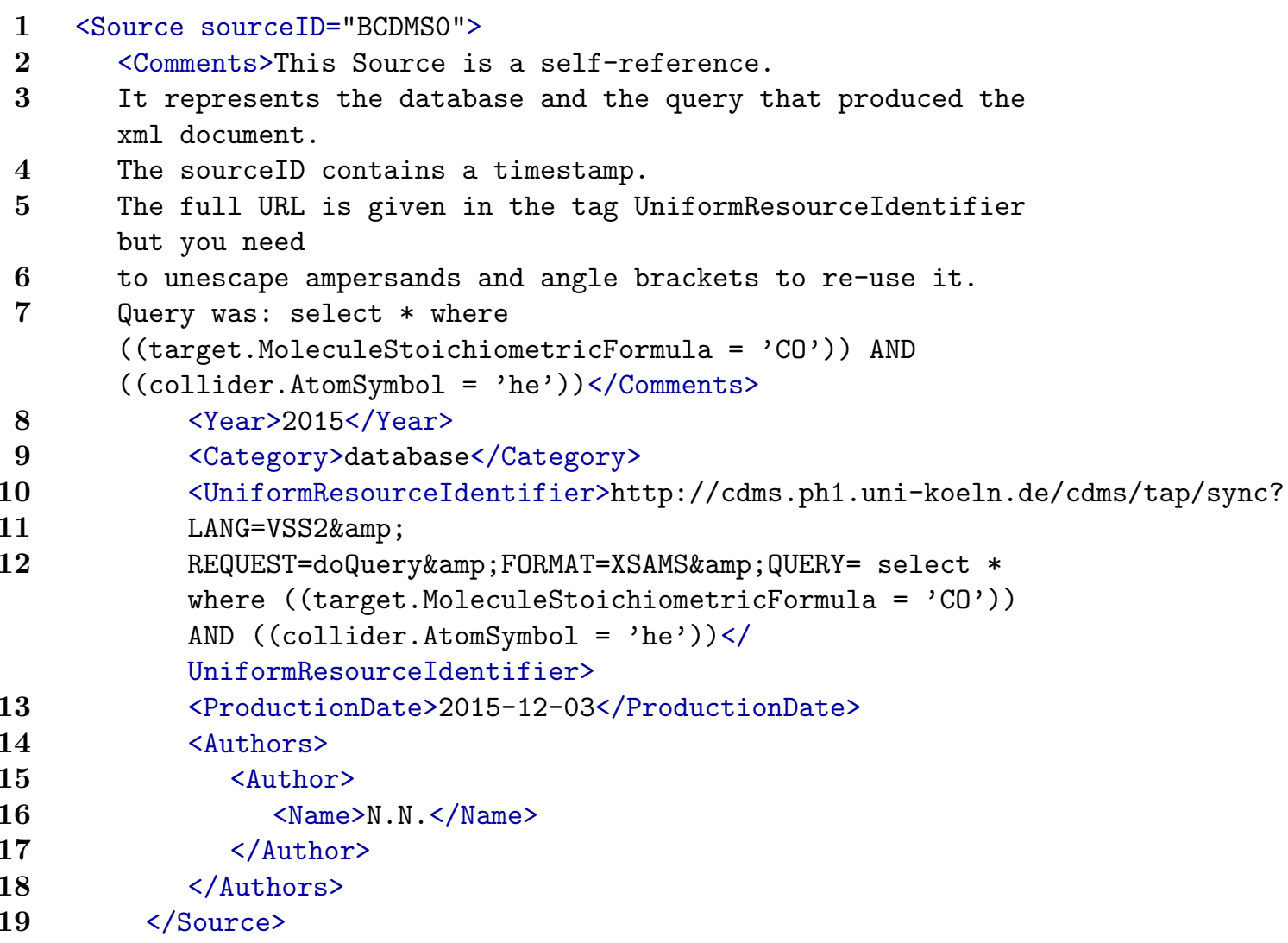

at line 150 of $B$

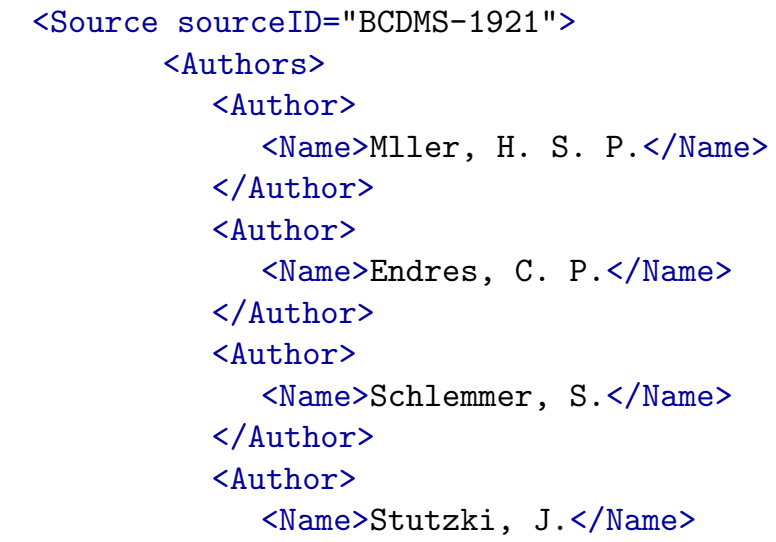




$\begin{array}{ll}\mathbf{1 4} & \text { </Author }> \\ \mathbf{1 5} & \text { </Authors } \\ \mathbf{1 6} & \text { <Title /> } \\ \mathbf{1 7} & \text { <Category>database</Category> } \\ \mathbf{1 8} & \text { <Year }>2012</ \text { Year }> \\ \mathbf{1 9} & \text { <SourceName>CDMS database</SourceName> } \\ \mathbf{2 0} & \text { </Source }>\end{array}$

and line 170 of $\mathrm{B}$

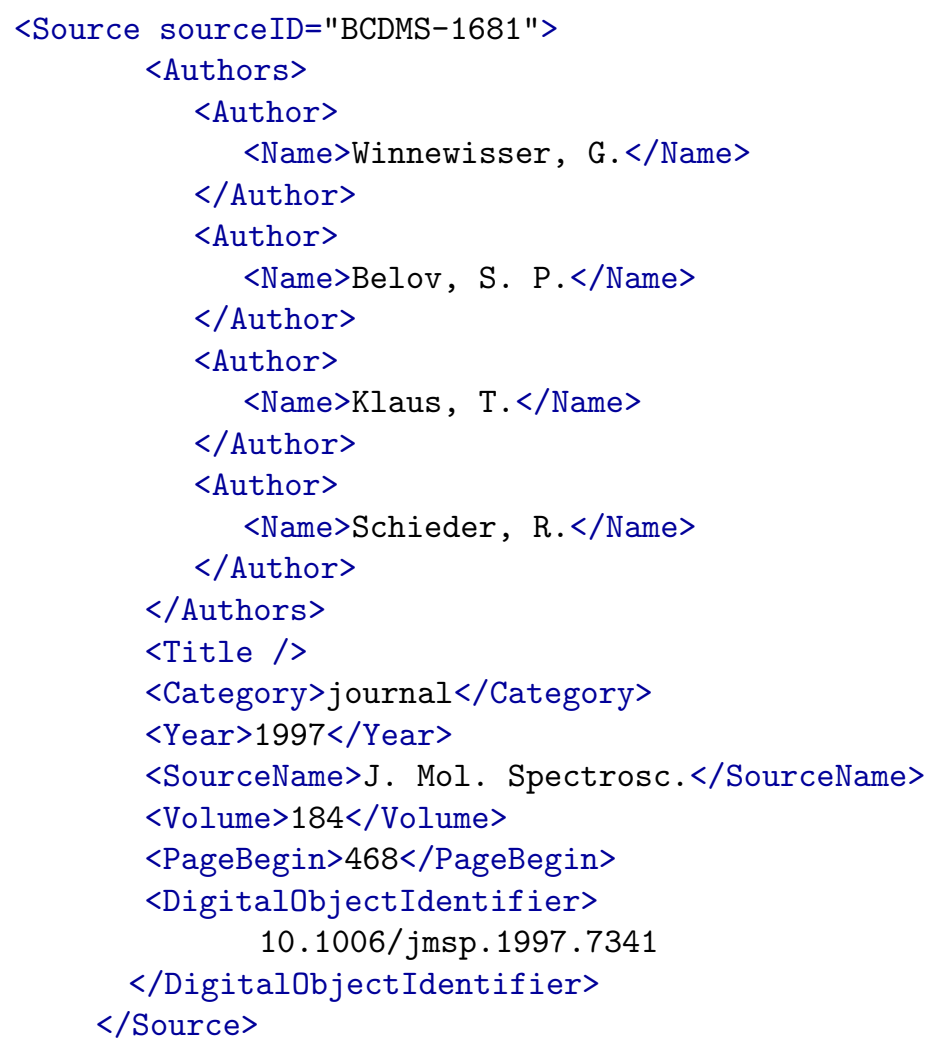

This last reference is [26].

\subsection{Documenting data processing workflow: merging of two files with SpectCol}

We used the SpectCol tool [3, $[7]^{2}$ for cross-matching the data from the two files described into the previous subparagraphs. The SpectCol tool allows to create combined spectroscopic and collisional data files where the collisional rate coefficients come from a collisional database (BASECOL in this example) and the spectroscopic data (energy levels and Einstein coefficients) come from a spectroscopic database (CDMS in this example). This process requires to match the spectroscopic energy levels of CDMS with those of

\footnotetext{
${ }^{2}$ http://www.vamdc.org/software/spectcol
} 
BASECOL on the basis of identical quantum numbers. We asked Spectcol to merge the two files by identifying the states of the Carbon Monoxyde characterised by the same quantum number values $J$ : the state referenced by the identifier SBASET52-1 ( $J=0$, cf. line 117 of A) has been identified and assimilated with $C D M S-83-1$ (cf. line 64 of $\mathrm{B}$ ) while the state referenced by the identifier SBASET52-2 ( $J=1$, cf. line 132 of A has been identified and assimilated with $C D M S-83-1$ (cf. line 80 of B).

The resulting merged file is reported in C. Let us browse the content of this file and focus on the root Origin element:

1

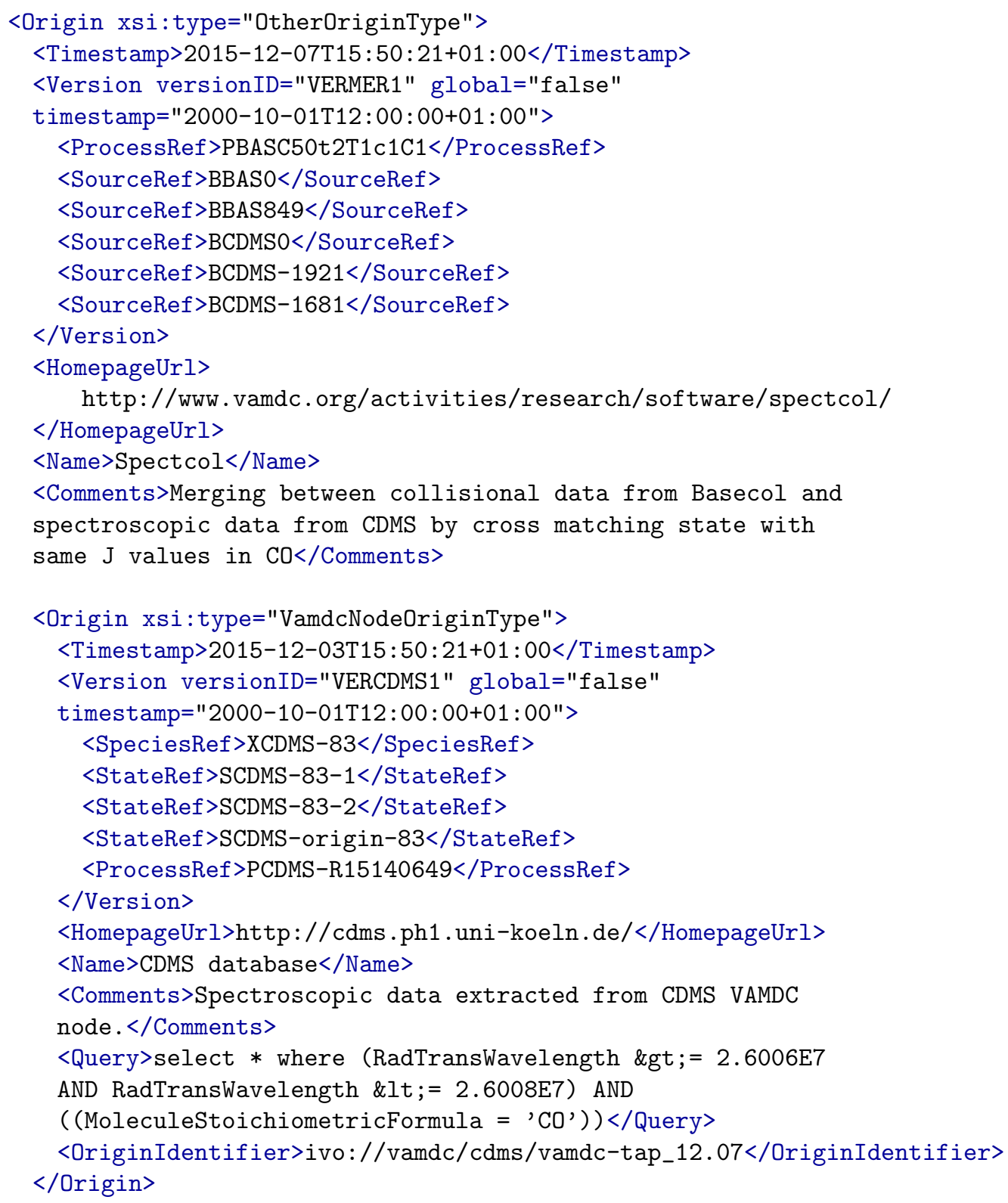




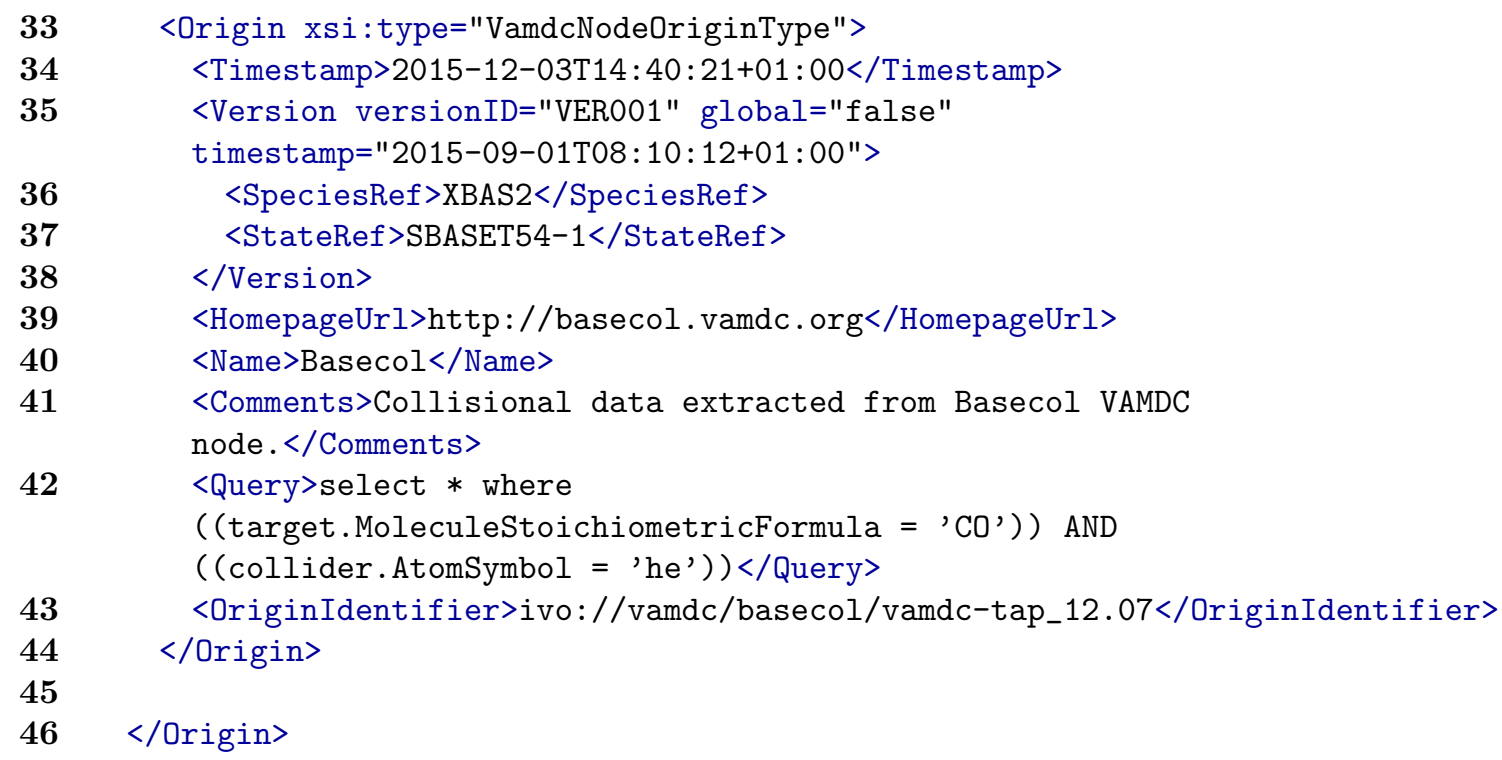

We see (line 1) that the file was not produced by a VAMDC node, but by the Spectcol tool (line 11 to 15). The current file has been produced on 2015-12-07T15:50:21+01:00 and results from the merging of data coming from BASECOL and from CDMS, where the states of $C O$ characterised by the same values of the quantum number $J$ are crossmatched (line 15). The data coming from BASECOL and CDMS are detailed into the two contained Origin sub-elements:

- the first one is defined from line 17 to line 31 and holds the data coming from the CDMS node. This includes the species referenced by the identifier $X C D M S-83$, the states referenced by the identifier SCDMS-83-1, SCDMS-83-2, SCDMS-origin-83 (respectively defined at line $137,151,165$ of C), the process referenced by the identifier $P C D M S-R 15140649$ (defined at line 186 of C). The extraction of those data was performed on 2015-12-03T15:50:21+01:00 from the CDMS Node by submitting the query

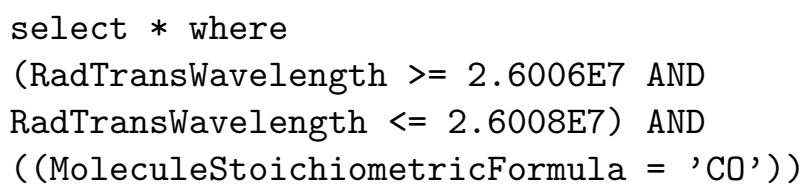

The extracted data correspond to the version VERCDMS1 that was available to the community since 2000-10-01T12:00:00+01:00

- the second one is defined from line 31 to line 42 and holds the data coming from the BASECOL node. This includes the species referenced by the identifier XBAS2 (defined at line 75 of $\mathrm{C}$ ) and the state referenced by the identifier SBASET54-1 (defined at line 77 of C). The extraction of those data was performed on 2015-12-03T14:40:21+01:00 
from the BASECOL node by submitting the query

select $*$ where

$(($ target.MoleculeStoichiometricFormula $=$ 'CO' $))$ AND

$(($ collider . AtomSymbol $=$ 'he' $))$

The extracted data correspond to the version VER001 of the database.

This was available to the community since 2015-09-01T08:10:12+01:00.

Let us focus again on the root Origin element:

- As we can see from line 5 to 9 , all the sources references from the merged files are now contained into the Version object of the root Origin. Indeed during the merging procedure, the sources from both the BASECOL and the CDMS files are compiled together into the root element since the data coming from all the sources are used. Moreover for data citation, it is very convenient to store at a same location all the references linked to the current file.

- The process referenced by PBASC50t2T1c1C1 belongs to the Version field of the root element (line 4). If we compare with A, line 154, we discover that this is a collisional process coming from BASECOL. It is important to understand why it is not contained into the second sub-origin element which, as we previously shown, contains the data coming from BASECOL: in its original extraction from BASECOL (cf. A, line 154) this process is a collision between $C O$ (identified with the label $X B A S 52)$ and $H e$ (identified with the label $X B A S 2)$. During the collision, the $C O$ pass from the state referenced by SBASET522 to the state referenced by SBASET52-1. All these identifiers and references have consistency in the BASECOL perimeter. Let us look at the same collisional process as it is described on the merged file (cf. line 212 of $\mathrm{C}$ ): the collision is between $C O$ (now identified with the label $X C D M S-83$, since during the crossmatching procedure we have replaced the $C O$ from BASECOL with the one coming from CDMS) and $H e$. During the collision $C O$ pass from the state referenced by $S C D M S-83-2$ to the state referenced by $S C D M S-83-1$ (indeed we remember that during the crossmatching we have identified the state $S B A S E T 52-2$ from BASECOL with the state $S C D M S-83-2$ from CDMS and the states SBASET52-1 from BASECOL with the state $S C D M S$-83-1 from CDMS). Due to these changes, the collisional process refer now to data coming both from BASECOL and CDMS and the data is no more the original one freshly extracted from BASECOL (as it was the case in A, line 154). As a direct consequence BASECOL is no more the source origin of this data. This combined collision is created by SpectCol and it is natural that it appears into the root Origin element which holds the data produced by SpectCol during the crossmatching procedure. 


\section{Practical use of the new XSAMS features}

In section 3 we introduced new changes and additions to the XSAMS format which allows one to identify unequivocally through time and location the datasets extracted from VAMDC: all the relevant metadata (the extraction query, the date when the query was submitted to the infrastructure, the responding node, the version of the responding node, etc...) are automatically embedded into the output file. But from the practical point of view of the final user, how to use this information? Our aim is to simplify the "data citation" landscape and not to complicate the actual one described in section 2. In this paragraph we are going to sketch the future VAMDC services that will allow a simple and fluid use of these new features:

When a user queries data from the VAMDC e-infrastructure by submitting a given query, he/she will receive an output file formatted as described in section 3. This file will be associated with a unique digital identifier. This identifier (as it is the case for the DOI), will be resolvable and the landing pag $\AA^{3}$ will summarize the information contained in the Origin field of the output file, the data-file itself (via query re-execution, if the node supports this feature) the bibtex references of all the papers used for compiling the output file. This sketched service is a part of a Query Store as it is understood in the Data Citation RDA context and described in [22].

This query store may be used in collaboration with editors. We imagine the following data-citation workflow: when a scientist wishes to cite a dataset extracted from VAMDC, he/she has just to put into the references the unique identifier returned by the VAMDC infrastructure during the extraction procedure. Since this unique identifier is resolvable, the editors may resolve it and, by accessing the bibtex information of the landing page, give credits to all the listed authors. This automatic credit delegation method may also work with current systems (e.g. the SAO/NASA Astrophysics Data System $[5$.

\footnotetext{
${ }^{3}$ The landing page will be human readable, but a machine actionable version may exist for automatic treatements

${ }^{4}$ The evolved XSAMS standard described in section 3 allows a data provider to univocally tag version of their data. Even if we strongly suggest that the VAMDC nodes give access (through the VAMC infrastructure) to all the subsequent versions of their data, we cannot enforce the data policy on the federated nodes. A given node may delete an old version when a new one is available. In that case the metadata policy will always allow the unambiguous identification of the extracted data, but data extraction re-execution will not be possible

${ }^{5}$ http://adswww.harvard.edu/
} 


\section{Concluding remarks}

Through this work we have discussed an evolution of the VAMDC standard output format which provides a mechanisms allowing to identify through time and location, in a sustainable and unique way, data sets extracted by users from the VAMDC e-infrastructure. With our proposed solution the information about the origin and version of data are embedded into (and indissoluble from) the file containing the atomic and molecular data. The same mechanisms define an unambiguous method for tracing and documenting the data processing workflows.

The methods and technical solutions introduced in this paper are designed for scientist wishing to efficiently cite the datasets and the dataproducers of the Atomic and Molecular data extracted from the VAMDC infrastructure:

let us consider an XSAMS data file containing $N$ references $(N>>1)$ to the sources used for the data file compilation.

Before the introduction of the solution discussed into this paper, a scientist wishing to publish a work based on that data file would need to include the $N$ publications in order to properly cite the data.

With the introduction of the proposed solution, the scientist will only cite the Digital Unique Identifier (DUI) returned by the VAMDC infrastructure during the extraction procedure. This DUI is resolvable and the landing page will contain the original query used for the generation of the data file and the bibliographic references to the $N$ publications.

\section{$7 \quad$ Acknowledgements}

Support for VAMDC has been provided through the VAMDC and the SUP@VAMDC projects funded under the "Combination of Collaborative Projects and Coordination and Support Actions" Funding Scheme of The Seventh Framework Program. Call topic: INFRA-2008-1.2.2 and INFRA-2012 Scientific Data Infrastructure. Grant Agreement numbers: 239108 and 313284. We acknowledge support from Paris Astronomical Data Center. We thank Thomas Marquart, Guy Rixon and Mickail Doronin from the VAMDC Consortium for fruitful discussions.

\section{A Sample output from BASECOL}

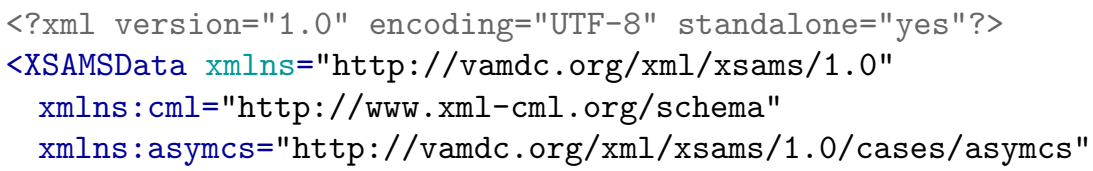


xmlns: asymos="http://vamdc .org/xml/xsams/1.0/cases/asymos"

xmlns:dcs="http://vamdc.org/xml/xsams/1.0/cases/dcs" xmlns: gen="http://vamdc . org/xml/xsams/1.0/cases/gen" xmlns: hunda="http://vamdc . org/xml/xsams/1.0/cases/hunda" xmlns: hundb="http://vamdc.org/xml/xsams/1.0/cases/hundb" xmlns: lpcs="http://vamdc.org/xml/xsams/1.0/cases/lpcs" xmlns: lpos="http://vamdc.org/xml/xsams/1.0/cases/lpos" xmlns:ltcs="http://vamdc.org/xml/xsams/1.0/cases/ltcs" xmlns: ltos="http://vamdc. org/xml/xsams/1.0/cases/ltos" xmlns:nltcs="http://vamdc .org/xml/xsams/1.0/cases/nltcs" xmlns:nltos="http://vamdc.org/xml/xsams/1.0/cases/nltos" xmlns: sphcs="http://vamdc. org/xml/xsams/1.0/cases/sphcs" xmlns: sphos="http://vamdc.org/xml/xsams/1.0/cases/sphos" xmlns: stcs="http://vamdc.org/xml/xsams/1.0/cases/stcs" xmlns: xsi="http://www . w3.org/2001/XMLSchema-instance" xsi: schemaLocation="http://vamdc.org/xml/xsams $/ 1$.0 https://raw.githubusercontent.com/nicolasmoreau/ VAMDC-XSAMS/abstract_origin/xsams.xsd">

<Origin xsi:type="VamdcNodeOriginType">

<Timestamp>2015-12-03T14:40:21+01:00</Timestamp>

<Version versionID="VER001" global="false"

timestamp $=" 2015-09-01 \mathrm{T08}: 10: 12+01: 00 ">$

$<$ SpeciesRef $>$ XBAS2</SpeciesRef $>$

$<$ SpeciesRef $>$ XBAS52</SpeciesRef $>$

$<$ StateRef $>$ SBASET54-1</StateRef $>$

$<$ StateRef $>$ SBASET52-1</StateRef $>$

$<$ StateRef $>$ SBASET52-2</StateRef $>$

$<$ ProcessRef $>$ PBASC50t2T1c1C1</ProcessRef>

$<$ SourceRef $>$ BBASO $</$ SourceRef $>$

$<$ SourceRef $>$ BBAS849</SourceRef $>$

$</$ Version $>$

<HomepageUrl>http://basecol.vamdc.org</HomepageUrl>

$<$ Name $>$ Basecol</Name>

$<$ Query>select $*$ where

( (target. MoleculeStoichiometricFormula = 'CO')) AND

$(($ collider.AtomSymbol $=$ 'he' $))</$ Query $\rangle$

<OriginIdentifier>ivo://vamdc/basecol/vamdc-tap_12.07</OriginIdentifier>

$</$ Origin $>$

$<$ Species $>$

$<$ Atoms $>$

$<$ Atom>

$<$ ChemicalElement>

$<$ NuclearCharge $>2</$ NuclearCharge $>$

$<$ ElementSymbol $>\mathrm{He}</$ ElementSymbol $>$

$</$ ChemicalElement $>$

<Isotope>

<IsotopeParameters>

$<$ MassNumber $>4</$ MassNumber $>$ 
<Mass>

$<$ Value units="amu" $>4.0026</$ Value $>$

</Mass $>$

$</$ IsotopeParameters $>$

<Ion speciesID="XBAS2">

$<$ IonCharge $>0</$ IonCharge $>$

<AtomicState stateID="SBASET54-1">

$<$ Comments>Energy level of $\mathrm{He}$ (no

structure) $<$ (Comments $>$

$<$ SourceRef $>$ BBASO $</$ SourceRef $>$

$<$ AtomicNumericalData>

<StateEnergy >

$<$ Value units $=" 1 / \mathrm{cm} ">0.0</$ Value $>$

$</$ StateEnergy $>$

$</$ AtomicNumericalData $>$

$<$ AtomicQuantumNumbers $>$

$<$ TotalAngularMomentum $>0.0</$ TotalAngularMomentum $>$

$</$ AtomicQuantumNumbers $>$

$<$ AtomicComposition>

<Component>

$<$ Term $>$

$<\mathrm{LS}>$

$<\mathrm{L}>$

$<$ Value $>0</$ Value $>$

$<$ Symbol $>$ L $</$ Symbol $>$

$</ \mathrm{L}>$

$\langle\mathrm{S}>0.0</ \mathrm{S}\rangle$

$</$ LS $>$

$</$ Term $>$

$</$ Component $>$

$</$ AtomicComposition $>$

$</$ AtomicState $>$

$<$ InChIKey>SWQJXJOGLNCZEY-UHFFFAOYSA-N</InChIKey> $</$ Ion $>$

$</$ Isotope $>$

$</$ Atom $>$

$</$ Atoms $>$

$<$ Molecules>

<Molecule speciesID="XBAS52">

<MolecularChemicalSpecies>

$<$ OrdinaryStructuralFormula>

$<$ Value $>$ CO $</$ Value $>$

$</$ OrdinaryStructuralFormula $>$

<StoichiometricFormula $>$ CO</StoichiometricFormula>

$<$ ChemicalName>

$<$ Value $>$ CO $</$ Value $>$

$</$ ChemicalName $>$

$<\operatorname{InChI}>\operatorname{InChI}=1 \mathrm{~S} / \mathrm{CO} / \mathrm{c} 1-2</ \mathrm{InChI}>$

$<$ InChIKey>UGFAIRIUMAVXCW-UHFFFAOYSA-N</InChIKey $>$ 
100

101

102

103

104

105

106

107

108

109

110

111

112

113

114

115

116

117

118

119

120

121

122

123

124

125

126

127

128

129

130

131

132

133

134

135

136

137

138

139

140

<VAMDCSpeciesID>UGFAIRIUMAVXCW-UHFFFAOYSA-N </VAMDCSpeciesID> <MoleculeStructure>

$<\mathrm{cml}$ : atomArray>

$<\mathrm{cml}$ : atom hydrogenCount="0" isotopeNumber="16"

count="1.0"

formalCharge="1" elementType="0" id="RBAS43N357"/>

$<\mathrm{cml}$ : atom hydrogenCount="0" isotopeNumber="12"

count="1.0"

formalCharge="-1" elementType="C" id="RBAS43N358"/>

$</ \mathrm{cml}$ : atomArray>

$<\mathrm{cml}$ : bondArray $>$

$<\mathrm{cml}$ : bond order="T" atomRefs2="RBAS43N357

RBAS43N358"/>

$</ \mathrm{cml}$ : bondArray $>$

$</$ MoleculeStructure $>$

$<$ StableMolecularProperties $>$

$<$ MolecularWeight>

$<$ Value units $="$ amu" $>27.99</$ Value $>$

$</$ MolecularWeight>

$</$ StableMolecularProperties $>$

$<$ Comment>Theoretical rotational energy levels of $\mathrm{CO}$

(Cecchi-Pestellini, 2002)</Comment>

$</$ MolecularChemicalSpecies $>$

$<$ MolecularState stateID="SBASET52-1">

$<$ SourceRef $>$ BBASO $</$ SourceRef $>$

$<$ Description $>$ Theoretical rotational energy levels of CO (Cecchi-Pestellini, 2002)</Description>

$<$ MolecularStateCharacterisation>

<StateEnergy energyOrigin="SBASET52-1">

$<$ Value units $=" 1 / \mathrm{cm} ">0.0</$ Value $>$

$</$ StateEnergy $>$

$</$ MolecularStateCharacterisation>

$<$ Case

xmlns:xsi="http://www.w3.org/2001/XMLSchema-instance"

xsi:type="dcs:Case" caseID="dcs">

<dcs:QNs>

$<$ dcs:ElecStateLabel>X/dcs:ElecStateLabel>

$\langle$ dcs : J $>0</$ des : J $>$

$</$ dcs:QNs $>$

$</$ Case $>$

$</$ MolecularState $>$

$<$ MolecularState stateID="SBASET52-2">

<SourceRef $>$ BBASO</SourceRef $>$

$<$ Description>Theoretical rotational energy levels of

CO (Cecchi-Pestellini, 2002)</Description>

<MolecularStateCharacterisation>

<StateEnergy energyOrigin="SBASET52-1">

$<$ Value units $=" 1 / \mathrm{cm} ">3.85</$ Value $>$

$</$ StateEnergy $>$ 
</MolecularStateCharacterisation> $<$ Case

xmlns: $x$ si="http://www.w3.org/2001/XMLSchema-instance" xsi:type="dcs:Case" caseID="dcs"> 
185

186

187

188

189

190

191

192

193

194

195

196

197

198

199

200

201

202

203

204

205

206

207

208

209

210

211

212

213

214

215

216

217

218

219

220

221

222

223

224

225

226

227

228

<DataList>3.4E-11 3.2E-11 3.0E-11 2.8E-11

$2.7 \mathrm{E}-11 \quad 2.6 \mathrm{E}-11 \quad 2.6 \mathrm{E}-11 \quad 2.5 \mathrm{E}-11 \quad 2.5 \mathrm{E}-11$

$2.6 \mathrm{E}-11</$ DataList $>$

$</ Y>$

$</$ TabulatedData $>$

$</$ DataSet $>$

$</$ DataSets $>$

$</$ CollisionalTransition $>$

$</$ Collisions $>$

$</$ Processes $>$

$<$ Sources>

<Source sourceID="BBASO">

<Category>database</Category>

<SourceName>BASECOL database</SourceName>

$<$ Year $>2015</$ Year $>$

$<$ Authors $>$

$<$ Author>

$<$ Name $>$ M. - L. Dubernet $</$ Name $>$

$</$ Author $>$

$</$ Authors $>$

<UniformResourceIdentifier>http://basecol.obspm.fr</UniformResourceIdentifier>

<ProductionDate>2015-12-03</ProductionDate>

$<$ Comments $>$ select $*$ where

( (target.MoleculeStoichiometricFormula = 'CO')) AND

$(($ collider. AtomSymbol $=$ 'he' $))\langle/$ Comments $\rangle$

$</$ Source $>$

$<$ Source sourceID="BBAS849">

$<$ Category $>$ journal</Category $>$

$<$ SourceName $>$ apj $</$ SourceName $>$

$<$ Year $>2002</$ Year $>$

$<$ Authors>

$<$ Author>

$<$ Name $>$ N. Balakrishnan</Name $>$

$</$ Author $>$

$<$ Author $>$

$<$ Name $>$ A. Dalgarno</Name $>$

$</$ Author $>$

$<$ Author $>$

$<$ Name $>$ C. Cecchi-Pestellini</Name $>$

$</$ Author $>$

$<$ Author $>$

$<$ Name $>$ E. Bodo</Name $>$

$</$ Author $>$

$</$ Authors $>$

$<$ Title>Rotational and Vibrational Excitation of CO

Molecules by Collisions with $\$^{\wedge}\{4\} \$$ He Atoms $</$ Title>

$<$ Volume $>571</$ Volume $>$

$<$ PageBegin $>1015</$ PageBegin $>$

$<$ PageEnd $>1020<$ /PageEnd $>$ 


\section{B Sample output from CDMS}

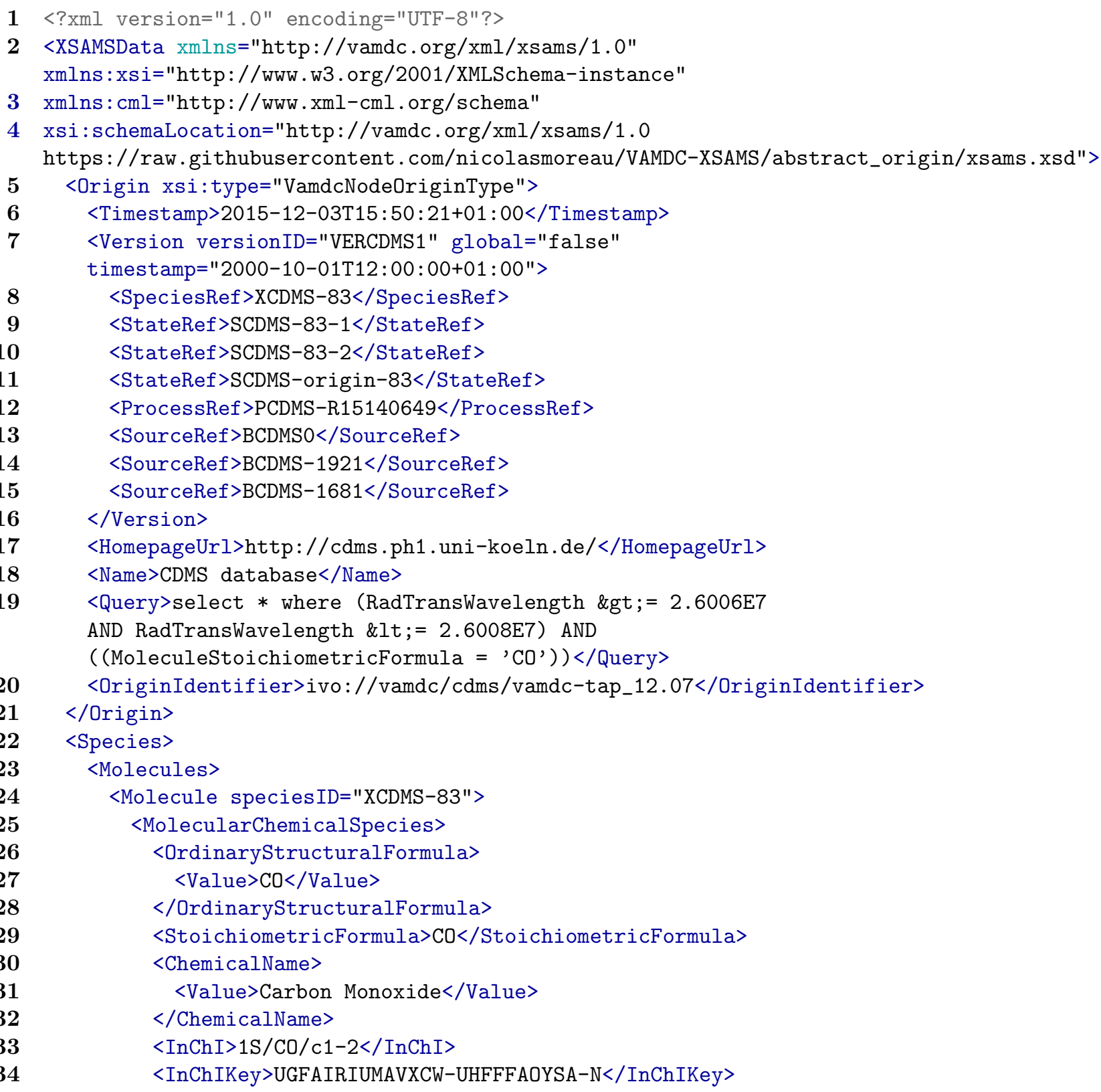


35

36

37

38

39

40

41

<VAMDCSpeciesID>UGFAIRIUMAVXCW-UHFFFAOYSA-N </VAMDCSpeciesID> $<$ PartitionFunction>

$<$ T units $=" \mathrm{~K} ">$

<DataList>1.072 $1.1481 .231 .3181 .413 \quad 1.514 \quad 1.622$

$\begin{array}{llllllll}1.738 & 1.862 & 1.995 & 2.138 & 2.291 & 2.455 & 2.63 & 2.725\end{array}$

$\begin{array}{llllllll}2.818 & 3.02 & 3.236 & 3.467 & 3.715 & 3.981 & 4.266 & 4.571\end{array}$

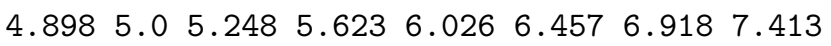

$\begin{array}{lllllllll}7.943 & 8.511 & 9.12 & 9.375 & 9.772 & 10.471 & 11.22 & 12.023\end{array}$

$\begin{array}{llllllll}12.882 & 13.804 & 14.791 & 15.849 & 16.982 & 18.197 & 18.75\end{array}$

$\begin{array}{llllllll}19.498 & 20.893 & 22.387 & 23.988 & 25.704 & 27.542 & 29.512\end{array}$

$31.623 \quad 33.88436 .30837 .5 \quad 38.905 \quad 41.68744 .668$

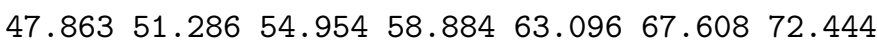

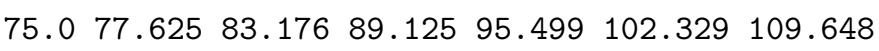

$117.49 \quad 125.893 \quad 134.896 \quad 144.544 \quad 150.0 \quad 154.882$

$\begin{array}{lllllll}165.959 & 177.828 & 190.546 & 204.174 & 218.776 & 225.0\end{array}$

$234.423 \quad 251.189269 .153 \quad 288.403 \quad 300.0 \quad 309.03$

$331.131 \quad 354.813 \quad 380.189407 .38 \quad 436.516 \quad 467.735$

$500.0 \quad 501.187 \quad 537.032575 .44616 .595 \quad 660.693$

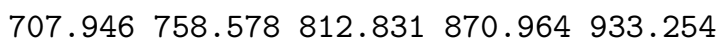

$1000.0</$ DataList $>$

$\langle/ \mathrm{T}\rangle$

$\langle Q\rangle$

<DataList>1.01721581835 1.02422837677

1.033411025221 .045121412451 .05985020123

$\begin{array}{llll}1.07774884294 & 1.09923417933 & 1.12473229226\end{array}$

$\begin{array}{llll}1.15441650589 & 1.18864057708 & 1.22774305695\end{array}$

1.271748642121 .320921247331 .37520246158

$1.40530654907 \quad 1.43514349042 \quad 1.50100121324$

$\begin{array}{llll}1.57270208471 & 1.65050158216 & 1.73501239088\end{array}$

$\begin{array}{llll}1.8265276783 & 1.92535019369 & 2.03179140659\end{array}$

$\begin{array}{llll}2.14652192999 & 2.18241666936 & 2.26987115366\end{array}$

$\begin{array}{llll}2.40252640741 & 2.54553752733 & 2.69889427601\end{array}$

$2.8632973732 \quad 3.04016627563 \quad 3.22985295285$

$3.4334258678 \quad 3.651956389493 .74352703447$

$3.88615863094 \quad 4.137466414184 .40695607053$

$4.69606494313 \quad 5.00551115749 \quad 5.33781506844$

$5.69369660756 \quad 6.07531823636 .48412210856$

$6.9226335574 \quad 7.122254331827 .3922960322$

$\begin{array}{ll}7.89599749116 \quad 8.4355434648 & 9.0138233311\end{array}$

$9.633727213 \quad 10.2977845092 \quad 11.0096090065$

$11.7724538098 \quad 12.5895724935 \quad 13.4656648032$

$\begin{array}{llll}13.8965040828 & 14.4043468795 & 15.4099582553\end{array}$

$16.4875620501 \quad 17.6425836137 \quad 18.8800875183$

20.206223673621 .627142876323 .1500815655

$24.7815541073 \quad 26.5302455525 \quad 27.4545173427$

$28.40375754 \quad 30.411139605932 .5625279126$

$\begin{array}{llll}34.8676989471 & 37.3378779761 & 39.9850161394\end{array}$

$42.8214291179 \quad 45.8608826253 \quad 49.1175078254$

$52.6076105394 \quad 54.581357915956 .3475015965$ 
$60.3549445917 \quad 64.6491571735 \quad 69.2508130864$

$74.1820459087 \quad 79.4660945815 \quad 81.7185083304$

$\begin{array}{llll}85.1287658147 & 91.1970158766 & 97.6997287418\end{array}$

$104.669260558 \quad 108.868854329 \quad 112.139428854$

$120.146852376 \quad 128.732781329 \quad 137.942190312$

$147.825233889158 .438549717 \quad 169.84604324$

$181.685035016 \quad 182.121688241 \quad 195.35029188$

209.62906021225 .070653126241 .803934912

259.976802963279 .755720344301 .328756805

$324.90561697350 .71803967379 .021079637</$ DataList $>$ $\langle/ Q\rangle$

$</$ PartitionFunction $>$

<StableMolecularProperties>

$<$ MolecularWeight>

$<$ Value units="unitless" $>28</$ Value $>$

$</$ MolecularWeight $>$

$</$ StableMolecularProperties $>$

<Comment> 28503- v 1:CO; $\$ \mathrm{v}=0 \$</$ Comment>

</MolecularChemicalSpecies>

$<$ MolecularState auxillary="true"

stateID="SCDMS-origin-83">

<MolecularStateCharacterisation>

<StateEnergy energyOrigin="SCDMS-origin-83">

$<$ Value units $=" 1 / \mathrm{cm} ">0.0</$ Value $>$

$</$ StateEnergy $>$

<TotalStatisticalWeight $>1</$ TotalStatisticalWeight $>$

$<$ NuclearStatisticalWeight $>1</$ NuclearStatisticalWeight $>$

$</$ MolecularStateCharacterisation $>$

$<$ Case

xmlns : dcs="http: //vamdc.org/xml/xsams/1.0/cases/dcs"

xsi:type="dcs:Case" caseID="dcs"

xsi:schemaLocation="http://vamdc .org/xml/xsams/1.0/cases/dcs

.././cases/dcs.xsd">

$\langle$ dcs:QNs>

<dcs: ElecStateLabel>X</dcs:ElecStateLabel>

$\langle$ dcs : v $>0</$ dcs : v $>$

$\langle$ dcs : J $>0</$ dcs : J $\rangle$

$\langle/$ dcs:QNs $>$

$</$ Case $>$

$</$ MolecularState $>$

<MolecularState stateID="SCDMS-83-1">

<MolecularStateCharacterisation>

<StateEnergy energyOrigin="SCDMS-origin-83">

$<$ Value units $=" 1 / \mathrm{cm} ">0.0</$ Value $>$

$</$ StateEnergy $>$

<TotalStatisticalWeight>1</TotalStatisticalWeight>

<NuclearStatisticalWeight $>1</$ NuclearStatisticalWeight $>$

$</$ MolecularStateCharacterisation $>$ 
xmlns:dcs="http://vamdc.org/xml/xsams/1.0/cases/dcs" xsi:type="dcs:Case" caseID="dcs"

xsi:schemaLocation="http://vamdc.org/xml/xsams/1.0/cases/dcs

.././cases/dcs.xsd"> <dcs:QNs>

<dcs:ElecStateLabel $>$ X</dcs:ElecStateLabel> $\langle$ dcs : v $>0</$ dcs : v $>$ 


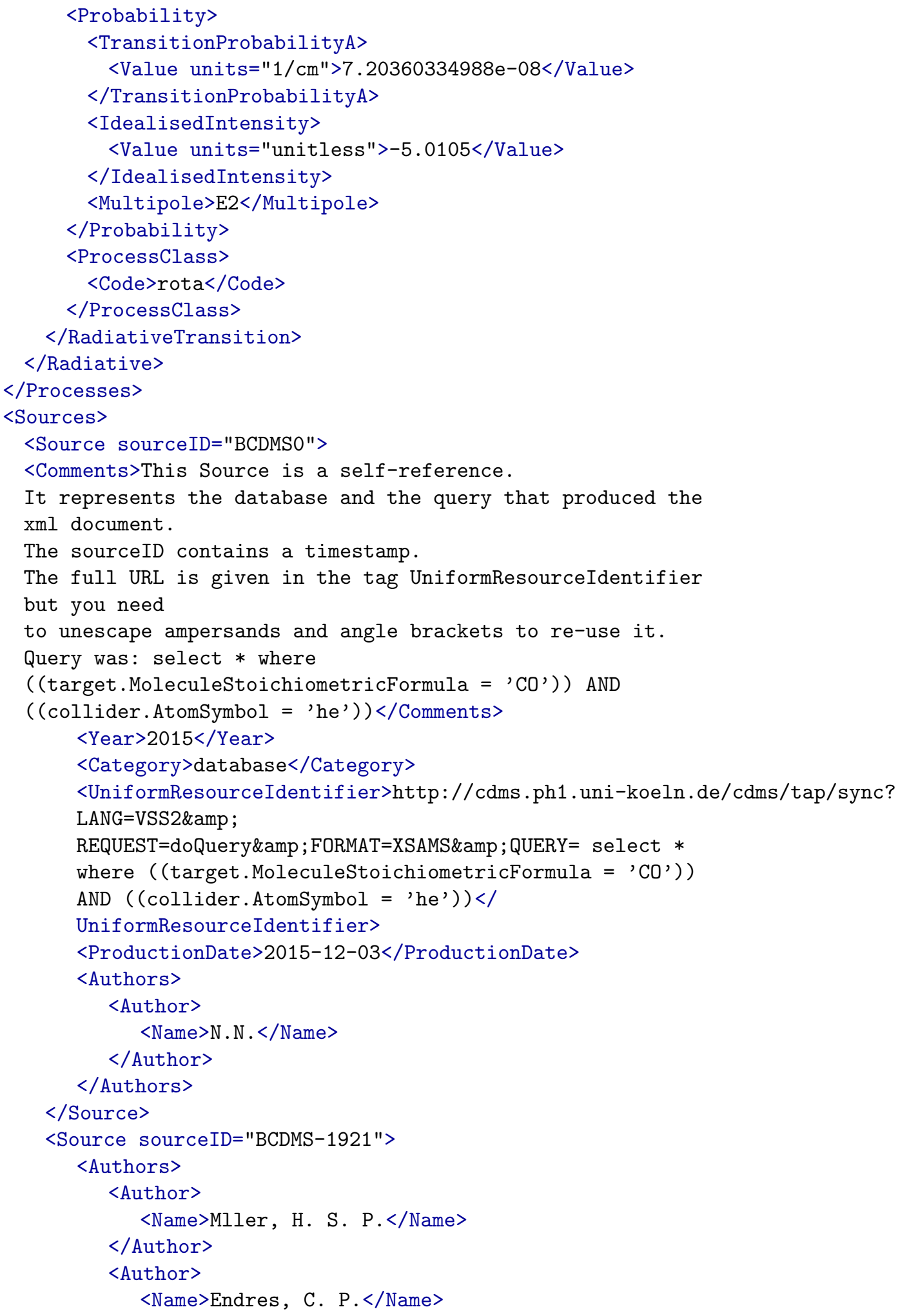




\section{Merged output from SpectCol}

</Author>

$<$ Author>

$<$ Name>Schlemmer, S. </Name>

$</$ Author $>$

$<$ Author $>$

$<$ Name $>$ Stutzki, J.</Name $>$

$</$ Author $>$

$</$ Authors $>$

$<$ Title / $>$

<Category>database</Category>

$<$ Year $>2012</$ Year $>$

$<$ Source $>$

<Source sourceID="BCDMS-1681" >

<Authors>

$<$ Author $>$

$<$ Name>Winnewisser, G.</Name>

$</$ Author $>$

$<$ Author $>$

$<$ Name $>$ Belov, S. P. $</$ Name $>$

$</$ Author $>$

$<$ Author $>$

$<$ Name>Klaus, T.</Name>

$</$ Author $>$

$<$ Author $>$

$<$ Name>Schieder, R. </Name>

$</$ Author $>$

$</$ Authors $>$

<Title />

$<$ Category $>$ journal</Category $>$

$<$ Year $>1997</$ Year $>$

$<$ Volume $>184</$ Volume $>$

$<$ PageBegin>468</PageBegin> $<$ Source $>$

$</$ Sources $>$

$</$ XSAMSData $>$
$<$ SourceName>CDMS database</SourceName>

$<$ SourceName>J. Mol. Spectrosc.</SourceName $>$

<DigitalObjectIdentifier>10.1006/jmsp.1997.7341</DigitalObjectIdentifier> 
xmlns: hundb="http://vamdc.org/xml/xsams/1.0/cases/hundb" xmlns: lpcs="http://vamdc.org/xml/xsams/1.0/cases/lpcs" xmlns: lpos="http://vamdc . org/xml/xsams/1.0/cases/lpos" xmlns: ltcs="http://vamdc.org/xml/xsams/1.0/cases/ltcs" xmlns: ltos="http://vamdc.org/xml/xsams/1.0/cases/ltos" xmlns:nltcs="http://vamdc .org/xml/xsams/1.0/cases/nltcs" xmlns:nltos="http://vamdc .org/xml/xsams/1.0/cases/nltos" xmlns: sphcs="http://vamdc.org/xml/xsams/1.0/cases/sphcs" xmlns: sphos="http://vamdc.org/xml/xsams/1.0/cases/sphos" xmlns: stcs="http://vamdc.org/xml/xsams/1.0/cases/stcs" xmlns:xsi="http://www.w3.org/2001/XMLSchema-instance" xsi:schemaLocation="http://vamdc.org/xml/xsams/1.0 https://raw.githubusercontent.com/nicolasmoreau/VAMDC-XSAMS/abstract_origin/xsams.xsd" $>$ 


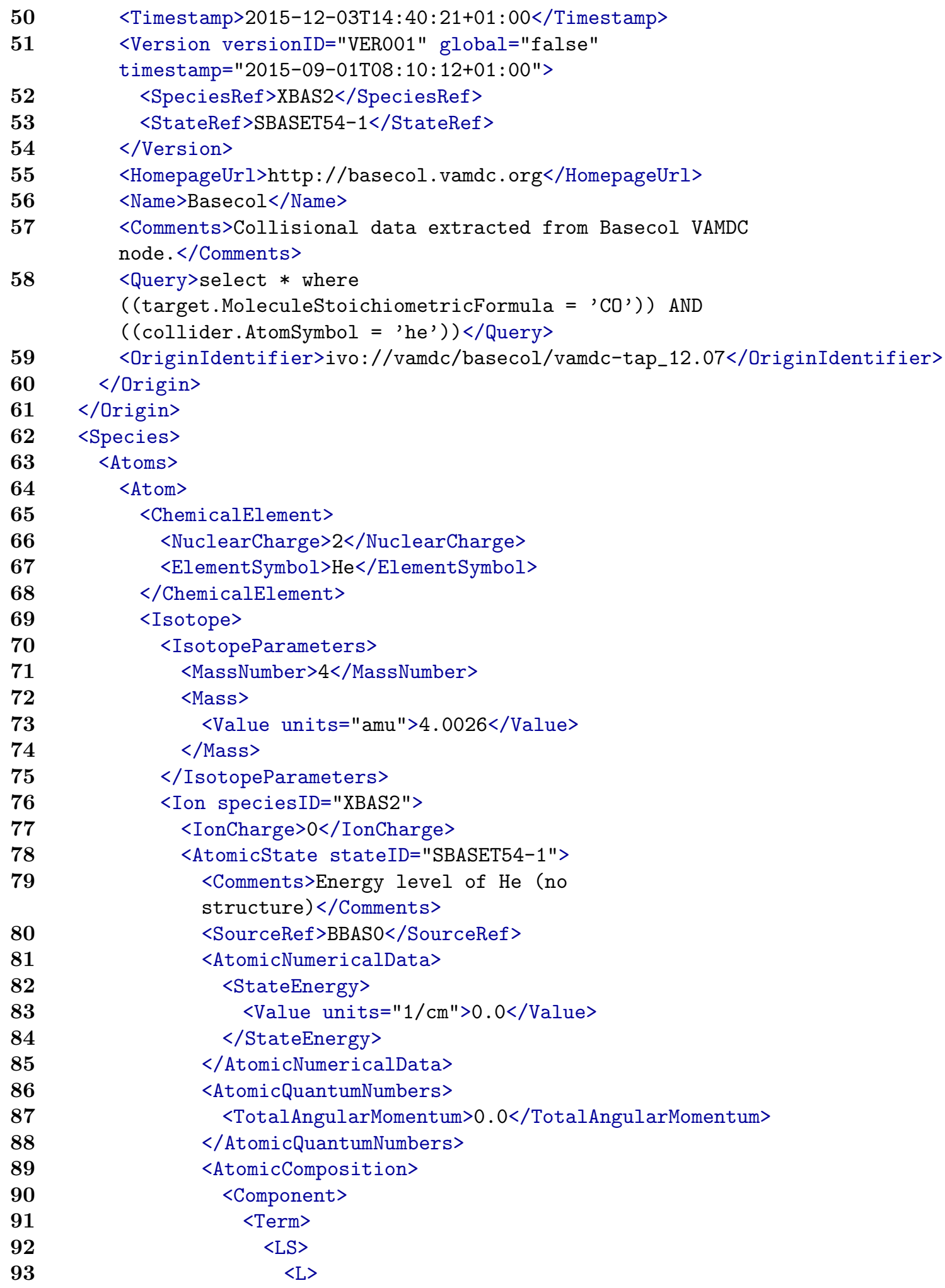

<Timestamp>2015-12-03T14:40:21+01:00</Timestamp> 


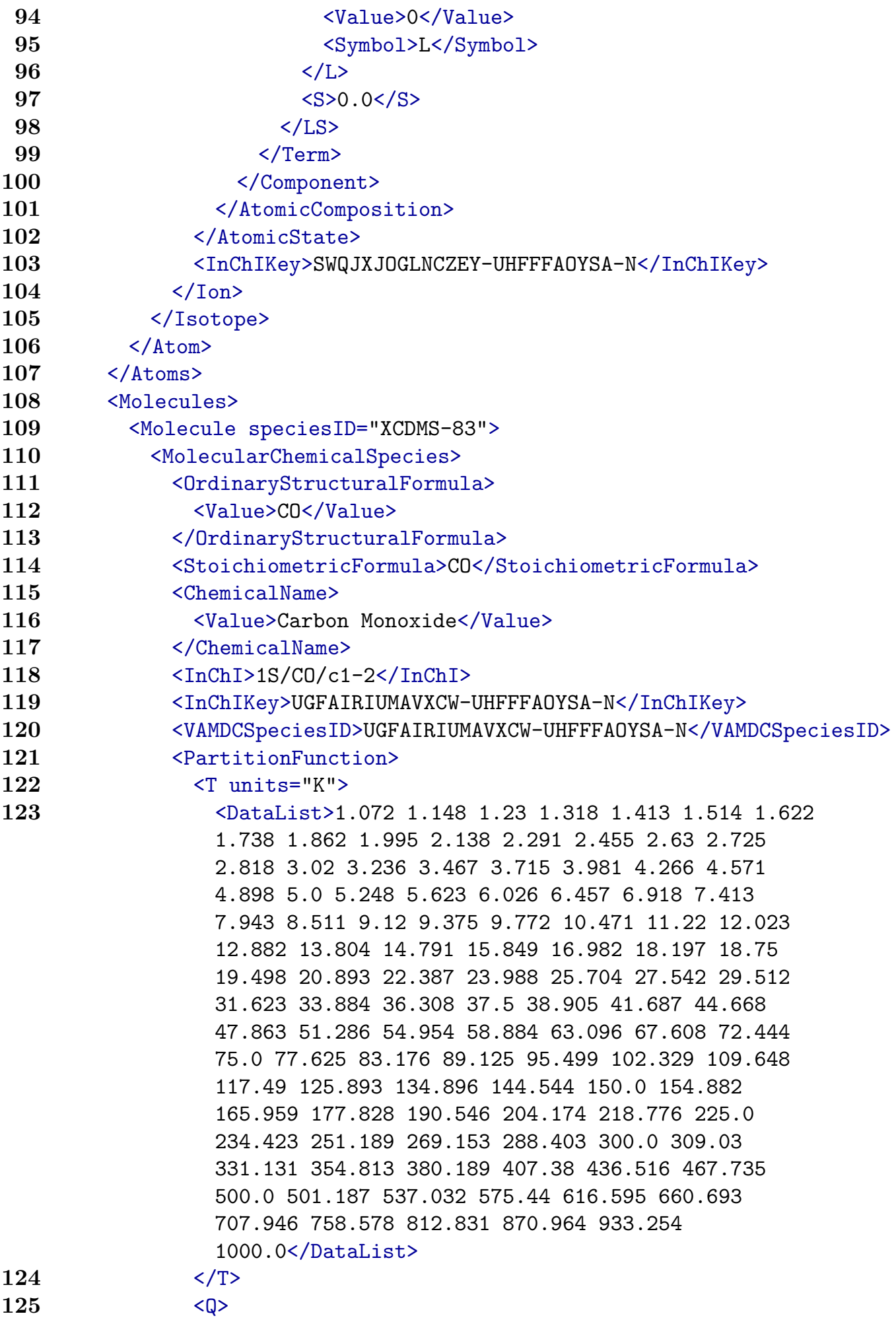


<DataList>1.01721581835 1.02422837677

$\begin{array}{llll}1.03341102522 & 1.04512141245 & 1.05985020123\end{array}$

$\begin{array}{llll}1.07774884294 & 1.09923417933 & 1.12473229226\end{array}$

$\begin{array}{llll}1.15441650589 & 1.18864057708 & 1.22774305695\end{array}$

$1.27174864212 \quad 1.32092124733 \quad 1.37520246158$

$1.40530654907 \quad 1.43514349042 \quad 1.50100121324$

$\begin{array}{llll}1.57270208471 & 1.65050158216 & 1.73501239088\end{array}$

$\begin{array}{llll}1.8265276783 & 1.92535019369 & 2.03179140659\end{array}$

$\begin{array}{llll}2.14652192999 & 2.18241666936 & 2.26987115366\end{array}$

$2.40252640741 \quad 2.54553752733 \quad 2.69889427601$

$2.8632973732 \quad 3.04016627563 \quad 3.22985295285$

$3.4334258678 \quad 3.651956389493 .74352703447$

$3.88615863094 \quad 4.13746641418 \quad 4.40695607053$

4.696064943135 .005511157495 .33781506844

$\begin{array}{llll}5.69369660756 & 6.0753182363 & 6.48412210856\end{array}$

$6.9226335574 \quad 7.122254331827 .3922960322$

$\begin{array}{llll}7.89599749116 & 8.4355434648 & 9.0138233311\end{array}$

$9.633727213 \quad 10.297784509211 .0096090065$

$11.7724538098 \quad 12.5895724935 \quad 13.4656648032$

$13.8965040828 \quad 14.4043468795 \quad 15.4099582553$

$\begin{array}{lllll}16.4875620501 & 17.6425836137 & 18.8800875183\end{array}$

$20.2062236736 \quad 21.6271428763 \quad 23.1500815655$

$24.7815541073 \quad 26.5302455525 \quad 27.4545173427$

$28.40375754 \quad 30.411139605932 .5625279126$

$\begin{array}{llll}34.8676989471 & 37.3378779761 & 39.9850161394\end{array}$

$42.8214291179 \quad 45.8608826253 \quad 49.1175078254$

$52.6076105394 \quad 54.5813579159 \quad 56.3475015965$

$60.3549445917 \quad 64.6491571735 \quad 69.2508130864$

$\begin{array}{lll}74.1820459087 & 79.4660945815 & 81.7185083304\end{array}$

$\begin{array}{llll}85.1287658147 & 91.1970158766 & 97.6997287418\end{array}$ $104.669260558 \quad 108.868854329 \quad 112.139428854$ $120.146852376 \quad 128.732781329 \quad 137.942190312$

$147.825233889158 .438549717 \quad 169.84604324$

$181.685035016182 .121688241 \quad 195.35029188$

209.62906021225 .070653126241 .803934912

259.976802963279 .755720344301 .328756805 $324.90561697350 .71803967379 .021079637</$ DataList $>$ $</ Q>$

$</$ PartitionFunction $>$

<StableMolecularProperties $>$

<MolecularWeight>

$<$ Value units="unitless" $>28.0</$ Value $>$

$</$ MolecularWeight $>$

$</$ StableMolecularProperties $>$

<Comment> 28503- v 1:CO; $\$ \mathrm{v}=0 \$</$ Comment $>$

$</$ MolecularChemicalSpecies>

<MolecularState stateID="SCDMS-83-1">

<MolecularStateCharacterisation>

<StateEnergy energyOrigin="SCDMS-origin-83" > 
$<$ Value units $=" 1 / \mathrm{cm} ">0.0</$ Value $>$

$</$ StateEnergy $>$

<TotalStatisticalWeight $>1</$ TotalStatisticalWeight $>$ <NuclearStatisticalWeight $>1</$ NuclearStatisticalWeight $>$ $<$ MolecularStateCharacterisation> $<$ Case xmlns: xsi="http://www . w3.org/2001/XMLSchema-instance" xsi:type="dcs:Case" caseID="dcs"> $<$ dcs:QNs> $\langle$ dcs : $\mathrm{J}\rangle 0</$ dcs : $\mathrm{J}\rangle$ $\langle/$ dcs : QNs $>$ $</$ Case $>$ $</$ MolecularState> <MolecularState stateID="SCDMS-83-2"> <MolecularStateCharacterisation> <StateEnergy energyOrigin="SCDMS-origin-83"> $<$ Value units $=" 1 / \mathrm{cm} ">3.845033</$ Value $>$ $</$ StateEnergy $>$ <TotalStatisticalWeight>3</TotalStatisticalWeight> <NuclearStatisticalWeight $>1</$ NuclearStatisticalWeight $>$ $</$ MolecularStateCharacterisation $>$ $<$ Case xmlns: xsi="http://www .w3.org/2001/XMLSchema-instance" xsi:type="dcs:Case" caseID="dcs"> $\langle$ dcs:QNs $>$ $\langle$ dcs : J $>1</$ des : J $\rangle$ $\langle/$ dcs:QNs $>$

$</$ Case $>$

$</$ MolecularState $>$

<MolecularState auxillary="true" stateID="SCDMS-origin-83">

$<$ MolecularStateCharacterisation> <StateEnergy energyOrigin="SCDMS-origin-83"> $<$ Value units $=" 1 / \mathrm{cm} ">0.0</$ Value $>$ $</$ StateEnergy $>$ <TotalStatisticalWeight $>1</$ TotalStatisticalWeight $>$ <NuclearStatisticalWeight $>1</$ NuclearStatisticalWeight $>$ $</$ MolecularStateCharacterisation $>$ $<$ Case xmlns:dcs="http: //vamdc.org/xml/xsams/1.0/cases/dcs" xsi:type="dcs:Case" caseID="dcs" xsi:schemaLocation="http://vamdc.org/xml/xsams/1.0/cases/dcs .././cases/dcs.xsd"> $\langle$ dcs:QNs> $<$ dcs:ElecStateLabel $>$ X</dcs:ElecStateLabel> $\langle$ dcs: $\mathrm{v}>0</$ dcs : $\mathrm{v}\rangle$ $<$ dc s : $\mathrm{J}>0</$ dc s : $\mathrm{J}\rangle$ $</$ dcs:QNs $>$

$</$ Case $>$ 


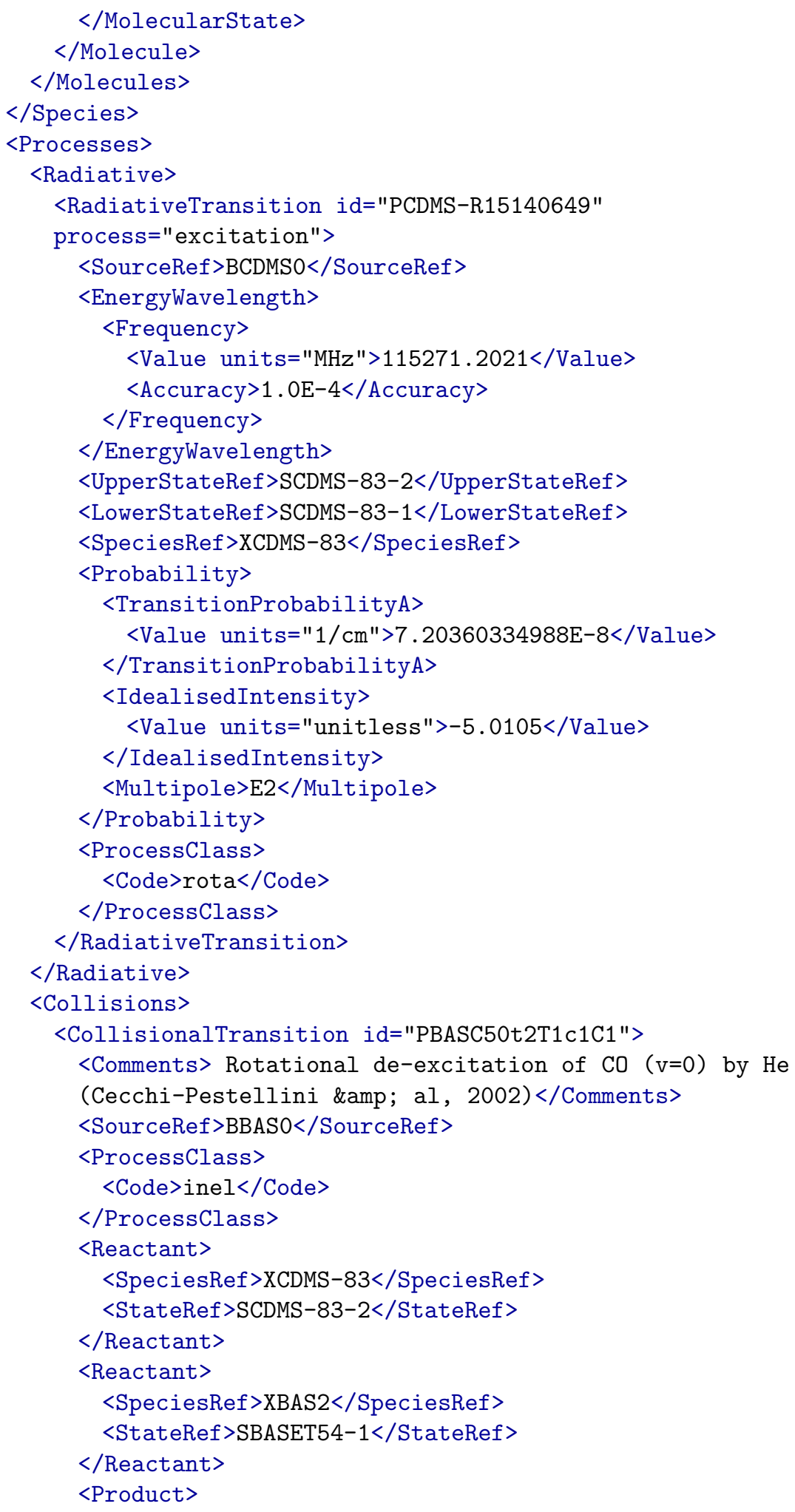




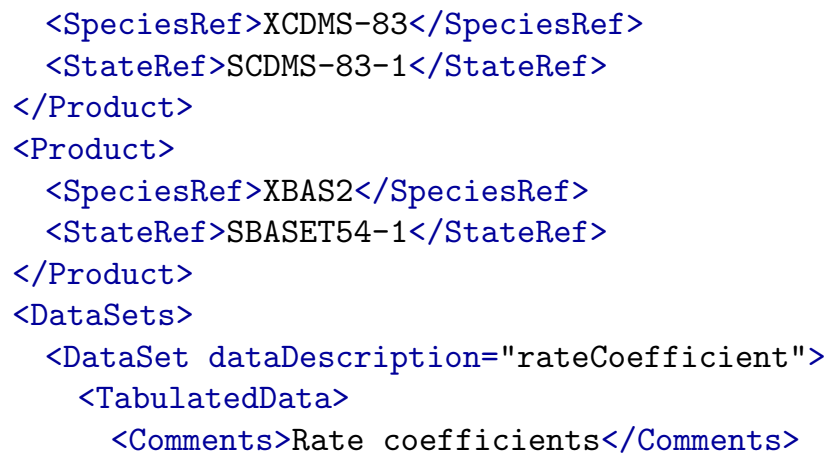


</Author>

<Author>

$<$ Name $>$ A. Dalgarno</Name $>$

$</$ Author $>$

$<$ Author>

$<$ Name>C. Cecchi-Pestellini</Name>

$</$ Author $>$

$<$ Author $>$

$<$ Name $>$ E. Bodo</Name $>$

$</$ Author $>$

$</$ Authors $>$

$<$ Title>Rotational and Vibrational Excitation of CO Molecules by Collisions with $\$^{\wedge}\{4\}$ He Atoms</Title>

$<$ Volume $>571</$ Volume $>$

$<$ PageBegin $>1015</$ PageBegin $>$

$<$ PageEnd $>1020</$ PageEnd $>$

<UniformResourceIdentifier>

http://adsabs.harvard.edu/cgi-bin/nph-bib_query? bibcode $=2002 \mathrm{JChPh} .116 .4517 \mathrm{~K} \& d b \_k e y=$ PHY

$</$ UniformResourceIdentifier $>$

$</$ Source $>$

$<$ Source sourceID="BCDMSO">

$<$ Comments>This Source is a self-reference.

It represents the database and the query that produced the xml document.

The sourceID contains a timestamp.

The full URL is given in the tag UniformResourceIdentifier but you need

to unescape ampersands and angle brackets to re-use it.

Query was: select * where

( (target.MoleculeStoichiometricFormula = 'CO')) AND

$(($ collider.AtomSymbol $=$ 'he' $))\langle/$ Comments $>$

$<$ Year $>2015</$ Year>

$<$ Category $>$ database $</$ Category $>$

<UniformResourceIdentifier>http://cdms.ph1.uni-koeln.de/cdms/tap/sync? LANG=VSS2\&amp;

REQUEST=doQuery\&amp; FORMAT=XSAMS\&amp; QUERY= select *

where ((target.MoleculeStoichiometricFormula = 'CO'))

AND $(($ collider. AtomSymbol $=$ 'he' $))</$

UniformResourceIdentifier $>$

<ProductionDate>2015-12-03</ProductionDate>

$<$ Authors $>$

$<$ Author>

$<$ Name $>$ N.N. $</$ Name $>$

$</$ Author $>$

$</$ Authors $>$

$</$ Source $>$

<Source sourceID="BCDMS-1921">

<Authors> 


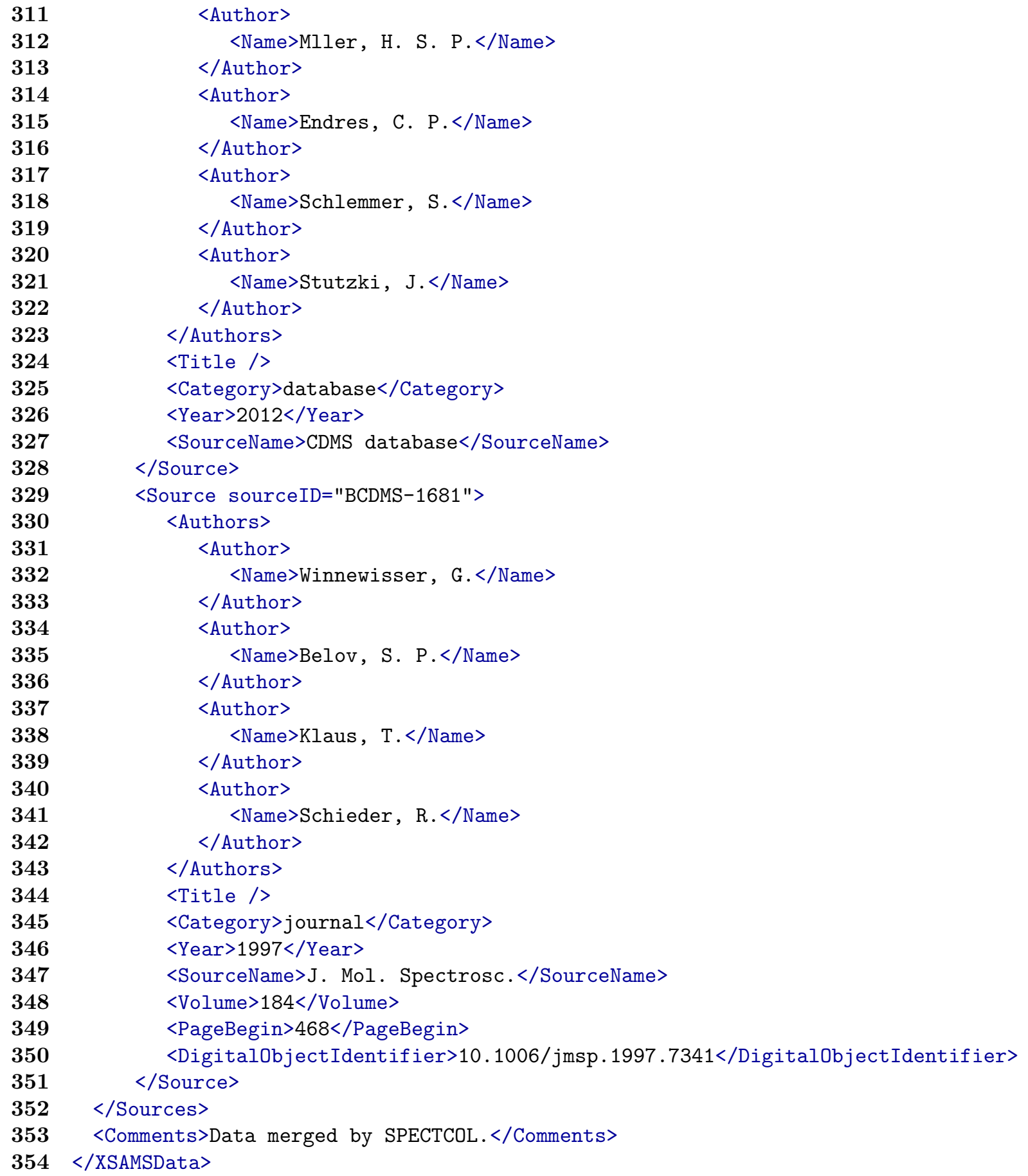

\section{References}

[1] Cesare Cecchi-Pestellini, Enrico Bodo, N. Balakrishnan, and Alexander Dalgarno. Rotational and vibrational excitation of co molecules 
by collisions with 4 he atoms. The Astrophysical Journal, 571(2):1015, 2002.

[2] J. F. Corby, P. A. Jones, M. R. Cunningham, K. M. Menten, A. Belloche, F. R. Schwab, A. J. Walsh, E. Balnozan, L. Bronfman, N. Lo, and A. J. Remijan. An ATCA survey of Sagittarius B2 at $7 \mathrm{~mm}$ : chemical complexity meets broad-band interferometry. M.N.R.A.S, 452:39693993, October 2015.

[3] M. Dubernet, L. Nenadovic, and N. Doronin. SPECTCOL: A New Software to Combine Spectroscopic Data and Collisional Data within VAMDC. In P. Ballester, D. Egret, and N. P. F. Lorente, editors, Astronomical Data Analysis Software and Systems XXI, volume 461 of Astronomical Society of the Pacific Conference Series, page 335, September 2012.

[4] M.-L. Dubernet, M. H. Alexander, Y. A. Ba, N. Balakrishnan, C. Balança, C. Ceccarelli, J. Cernicharo, F. Daniel, F. Dayou, M. Doronin, F. Dumouchel, A. Faure, N. Feautrier, D. R. Flower, A. Grosjean, P. Halvick, J. Kłos, F. Lique, G. C. McBane, S. Marinakis, N. Moreau, R. Moszynski, D. A. Neufeld, E. Roueff, P. Schilke, A. Spielfiedel, P. C. Stancil, T. Stoecklin, J. Tennyson, B. Yang, A.-M. Vasserot, and L. Wiesenfeld. BASECOL2012: A collisional database repository and web service within the Virtual Atomic and Molecular Data Centre (VAMDC). Astron. \& Astrophys., 553:A50, May 2013.

[5] M. L. Dubernet, V. Boudon, J. L. Culhane, M. S. Dimitrijevic, A. Z. Fazliev, C. Joblin, F. Kupka, G. Leto, P. Le Sidaner, P. A. Loboda, H. E. Mason, N. J. Mason, C. Mendoza, G. Mulas, T. J. Millar, L. A. Nuñez, V. I. Perevalov, N. Piskunov, Y. Ralchenko, G. Rixon, L. S. Rothman, E. Roueff, T. A. Ryabchikova, A. Ryabtsev, S. Sahal-Bréchot, B. Schmitt, S. Schlemmer, J. Tennyson, V. G. Tyuterev, N. A. Walton, V. Wakelam, and C. J. Zeippen. Virtual atomic and molecular data centre. J. Quant. Spectrosc. \& Rad. Transfer, 111:2151-2159, October 2010.

[6] M.-L. Dubernet, A. Grosjean, D. Flower, E. Roueff, F. Daniel, N. Moreau, and B. Debray. Ro-Vibrational Collisional Excitation Database: BASECOL - http://www.obspm.fr/basecol. Journal of Plasma Research SERIES, 7:356-357, January 2006.

[7] M.-L. Dubernet and L. Nenadovic. SPECTCOL: Spectroscopic and Collisional Data Retrieval. Astrophysics Source Code Library, November 2011.

[8] Marie-Lise Dubernet, Bobby Antony, Yaye-Awa Ba, Yuri Babikov, Klaus Bartschat, Vincent Boudon, B Braams, Hyun-Kyung Chung, 
Fabien Daniel, Franck Delahaye, Giulio Del Zanna, Jaime de Urquijo, Milan Dimitrijevic, Alicja Domaracka, Mikhail Doronin, Brian Drouin, Christian Endres, Alexandr Fazliev, Serguey Gagarin, Iouli Gordon, Pierre Gratier, Ulrike Heiter, Christian Hill, Darko Jevremovic, Christine Joblin, Aurlie Karsprzak, E. Krishnakumar, Giuseppe Leto, Peter A. Loboda, Thierry Louge, Sylvain Maclot, Bratislav Marinkovic, Andrew Markwick Kemper, Thomas Marquart, Helen Mason, Nigel Mason, Claudio Mendoza, Anatoly Mihajlov, Tom Millar, Nicolas Moreau, Giacomo Mulas, Yury Pakhomov, Patrick Palmeri, Sergey Pancheshnyi, Valery I. Perevalov, Nikolai Piskunov, Johannes Postler, Ernesto Luis Quinet, Pascaland Quintas Snchez, Yuri Ralchenko, YongJoo Rhee, Guy Rixon, Laurence Rothman, Evelyne Roueff, Tatiana Ryabchikova, Sylvie Sahal-Brechot, Paul Scheier, Stephan Schlemmer, Bernard Schmitt, Eric Stempels, Serguey Tashkun, Jonathan Tennyson, Vladimir Tyuterev, Veljko Vujcic, Valentine Wakelam, Nicolas Walton, Oleg Zatsarinny, Claude Zeippen, and Carlo Maria Zwölf. The Virtual Atomic and Molecular Data Centre (VAMDC) Consortium. Journal of Physics B: Atomic, Molecular and Optical Physics, 49(7):074003, 2016.

[9] M. A. Frerking and W. D. Langer. A measurement of the hyperfine structure of CO-17. J. Chem. Phys., 74:6990, June 1981.

[10] Ginard, D., Gonzlez-Garca, M., Fuente, A., Cernicharo, J., AlonsoAlbi, T., Pilleri, P., Gerin, M., Garca-Burillo, S., Ossenkopf, V., Rizzo, J. R., Kramer, C., Goicoechea, J. R., Pety, J., Bern, O., and Joblin, C. Spectral line survey of the ultracompact hii region monoceros $\mathrm{r} 2$ ? Astron. \& Astrophys., 543:A27, 2012.

[11] N. Huijboom and T. Van den Broek. Open data: an international comparison of strategies. European Journal of ePractice, N. 12, MarchApril 2011 - ISSN: 1988-625X, 2011.

[12] N. Jacquinet-Husson, L. Crepeau, R. Armante, C. Boutammine, A. Chédin, N. A. Scott, C. Crevoisier, V. Capelle, C. Boone, N. PouletCrovisier, A. Barbe, A. Campargue, D. C. Benner, Y. Benilan, B. Bézard, V. Boudon, L. R. Brown, L. H. Coudert, A. Coustenis, V. Dana, V. M. Devi, S. Fally, A. Fayt, J.-M. Flaud, A. Goldman, M. Herman, G. J. Harris, D. Jacquemart, A. Jolly, I. Kleiner, A. Kleinböhl, F. Kwabia-Tchana, N. Lavrentieva, N. Lacome, L.-H. Xu, O. M. Lyulin, J.-Y. Mandin, A. Maki, S. Mikhailenko, C. E. Miller, T. Mishina, N. Moazzen-Ahmadi, H. S. P. Müller, A. Nikitin, J. Orphal, V. Perevalov, A. Perrin, D. T. Petkie, A. Predoi-Cross, C. P. Rinsland, J. J. Remedios, M. Rotger, M. A. H. Smith, K. Sung, S. Tashkun, J. Tennyson, R. A. Toth, A.-C. Vandaele, and J. Vander Auwera. The 
2009 edition of the GEISA spectroscopic database. J. Quant. Spectrosc. E Rad. Transfer, 112:2395-2445, November 2011.

[13] N. Jacquinet-Husson, N. A. Scott, A. Chedin, B. Bonnet, A. Barbe, V. G. Tyuterev, J. P. Champion, M. Winnewisser, L. R. Brown, R. Gamache, V. F. Golovko, and A. A. Chursin. The GEISA system in 1996: towards an operational tool for the second generation vertical sounders radiance simulation. J. Quant. Spectrosc. \& Rad. Transfer, 59:511-527, May 1998.

[14] JC. Molloy. The Open Knowledge Foundation: Open Data Means Better Science. PLoS Biol 9(12): e1001195, 2011.

[15] H. S. P. Müller, F. Schlöder, J. Stutzki, and G. Winnewisser. The Cologne Database for Molecular Spectroscopy, CDMS: a useful tool for astronomers and spectroscopists. Journal of Molecular Structure, 742:215-227, May 2005.

[16] H. S. P. Müller, S. Thorwirth, D. A. Roth, and G. Winnewisser. The Cologne Database for Molecular Spectroscopy, CDMS. Astron. \& Astrophys., 370:L49-L52, April 2001.

[17] L. Pagani, F. Daniel, and M.-L. Dubernet. On the frequency of $\mathrm{N}\left\{{ }_{-} 2\right\} \mathrm{H}^{+}$and $\mathrm{N}\left\{\_2\right\} \mathrm{D}\left\{{ }^{\wedge}+\right\}$. Astron. $\&$ Astrophys., 494:719-727, February 2009 .

[18] H. M. Pickett, R. L. Poynter, E. A. Cohen, M. L. Delitsky, J. C. Pearson, and H. S. P. Müller. Submillimeter, millimeter and microwave spectral line catalog. J. Quant. Spectrosc. E Rad. Transfer, 60:883890, November 1998.

[19] N. E. Piskunov, F. Kupka, T. A. Ryabchikova, W. W. Weiss, and C. S. Jeffery. VALD: The Vienna Atomic Line Data Base. Astron. Astrophys. Suppl. Ser., 112:525, September 1995.

[20] HA. Piwowar and TJ. Vision. Data reuse and the open data citation advantage. ed. PeerJ. 2013;1:e175, 2013.

[21] A. Punanova, P. Caselli, A. Pon, A. Belloche, and P. André. Deuterium fractionation in the Ophiuchus molecular cloud. Astron. \&3 Astrophys., 587:A118, March 2016.

[22] Andreas Rauber, Ari Asmi, Dieter van Uytvanck, and Stefan Proell. Data Citation of Evolving Data - Recommendations of the Working Group on Data Citation. https://rd-alliance.org/rda-wgdcrecommendations-vers-sep-24-2015.html, September 2015. Draft - Request for Comments. 
[23] L. S. Rothman. Atmospheric absorption-line parameters: the HITRAN data base (A). Journal of the Optical Society of America (1917-1983), 73, 1983.

[24] L. S. Rothman, I. E. Gordon, Y. Babikov, A. Barbe, D. Chris Benner, P. F. Bernath, M. Birk, L. Bizzocchi, V. Boudon, L. R. Brown, A. Campargue, K. Chance, E. A. Cohen, L. H. Coudert, V. M. Devi, B. J. Drouin, A. Fayt, J.-M. Flaud, R. R. Gamache, J. J. Harrison, J.-M. Hartmann, C. Hill, J. T. Hodges, D. Jacquemart, A. Jolly, J. Lamouroux, R. J. Le Roy, G. Li, D. A. Long, O. M. Lyulin, C. J. Mackie, S. T. Massie, S. Mikhailenko, H. S. P. Müller, O. V. Naumenko, A. V. Nikitin, J. Orphal, V. Perevalov, A. Perrin, E. R. Polovtseva, C. Richard, M. A. H. Smith, E. Starikova, K. Sung, S. Tashkun, J. Tennyson, G. C. Toon, V. G. Tyuterev, and G. Wagner. The HITRAN2012 molecular spectroscopic database. J. Quant. Spectrosc. \& Rad. Transfer, 130:4-50, November 2013.

[25] T. Ryabchikova, N. Piskunov, R. L. Kurucz, H. C. Stempels, U. Heiter, Y. Pakhomov, and P. S. Barklem. A major upgrade of the VALD database. Physica Scripta, 90(5):054005, May 2015.

[26] G. Winnewisser, S. P. Belov, T. Klaus, and R. Schieder. Sub-Doppler Measurements on the Rotational Transitions of Carbon Monoxide. Journal of Molecular Spectroscopy, 184:468-472, August 1997.

[27] C.M. Zwolf, M.-L. Dubernet, Y.-A. Ba, and N. Moreau. Experience and feedbacks from the sustainability for the virtual atomic and molecular data centre e-infrastructure. In IST-Africa Conference Proceedings, 2014, pages 1-9, May 2014. 